\title{
Interactive 3D Space Group Visualization with CLUCalc and the Clifford Geometric Algebra De- scription of Space Groups
}

\author{
Eckhard Hitzer and Christian Perwass
}

Soli Deo Gloria

\begin{abstract}
A new interactive software tool is described, that visualizes 3D space group symmetries. The software computes with Clifford (geometric) algebra. The space group visualizer (SGV) originated as a script for the open source visual CLUCalc, which fully supports geometric algebra computation.

Selected generators (Hestenes and Holt, JMP, 2007) form a multivector generator basis of each space group. The approach corresponds to an algebraic implementation of groups generated by reflections (Coxeter and Moser, 4th ed., 1980). The basic operation is the reflection. Two reflections at non-parallel planes yield a rotation, two reflections at parallel planes a translation, etc. Combination of reflections corresponds to the geometric product of vectors describing the individual reflection planes.

We first give some insights into the Clifford geometric algebra description of space groups. We relate the choice of symmetry vectors and the origin of cells in the geometric algebra description and its implementation in the SGV to the conventional crystal cell choices in the International Tables of Crystallography (T. Hahn, Springer, 2005). Finally we briefly explain how to use the SGV beginning with space group selection. The interactive computer graphics can be used to fully understand how reflections combine to generate all 230 three-dimensional space groups.
\end{abstract}

Mathematics Subject Classification (2000). Primary 20H15; Secondary 15A66, 74N05, 76M27, 20F55 .

Keywords. Clifford geometric algebra, interactive software, space groups, crystallography, visualization. 


\section{Introduction}

Crystals are fundamentally periodic geometric arrangements of molecules (atoms or ions). The directed distance between two such molecules is a Euclidean vector in $\mathbb{R}^{3}$. Intuitively all symmetry properties of crystals depend on these vectors. Indeed, the geometric product of vectors [7] combined with the conformal model of three-dimensional (3D) Euclidean space $[1,8,15,17,18,27]$ yield an algebra fully expressing crystal point and space groups [10,13,19-21]. Two successive reflections at (non-) parallel planes express (rotations) translations, etc. [4,5] This leads to a 1:1 correspondence of geometric objects and symmetry operators with vectors and their products (Lipschitz elements, called versors in [13]), ideal for creating a suit of interactive visualizations using CLUCalc [24, 25] and OpenGL [19-21].

We begin by explaining the representation of point and space groups in conformal geometric algebra (sections 2 to 5). Then we relate the geometric algebra Bravais lattice symmetry vectors [14] and origins to the conventional crystallographic cell vectors and origin choices of [11] (section 6). Finally we explain the basic functions of the software implementation, called Space Group Visualizer (SGV) [26] in section 7.

\section{Cartan-Dieudonné and geometric algebra}

Clifford's associative geometric product [7] of two vectors simply adds the inner product to the outer product of Grassmann

$$
\boldsymbol{a b}=\boldsymbol{a} \cdot \boldsymbol{b}+\boldsymbol{a} \wedge \boldsymbol{b} .
$$

Under this product parallel vectors commute and perpendicular vectors anti-commute

$$
a x_{\|}=x_{\|} a, \quad a x_{\perp}=-x_{\perp} a .
$$

This allows to write the reflection of a vector $\boldsymbol{x}$ at a hyperplane ${ }^{1}$ through the origin with normal $\boldsymbol{a}$ as (see left side of Fig. 1)

$$
x^{\prime}=-a^{-1} x a, \quad a^{-1}=\frac{a}{a^{2}} .
$$

The composition of two reflections at hyperplanes, whose normal vectors $\boldsymbol{a}, \boldsymbol{b}$ subtend the angle $\alpha / 2$, yields a rotation around the intersection of the two hyperplanes (see right side of Fig. 1) by $\alpha$

$$
\boldsymbol{x}^{\prime \prime}=(\boldsymbol{a} \boldsymbol{b})^{-1} \boldsymbol{x} \boldsymbol{a} \boldsymbol{b}, \quad(\boldsymbol{a} \boldsymbol{b})^{-1}=\boldsymbol{b}^{-1} \boldsymbol{a}^{-1} .
$$

Continuing with a third reflection at a hyperplane with normal $c$ according to the CartanDieudonné theorem [2, 3,6,9] yields rotary reflections (equivalent to rotary inversions with angle $\alpha-\pi$ ) and inversions

$$
\boldsymbol{x}^{\prime}=-(\boldsymbol{a b c})^{-1} \boldsymbol{x} \boldsymbol{a b c}, \quad \boldsymbol{x}^{\prime \prime}=-i^{-1} \boldsymbol{x} i, \quad i \doteq \boldsymbol{a} \wedge \boldsymbol{b} \wedge \boldsymbol{c},
$$

\footnotetext{
${ }^{1}$ The corresponding matrix representation is known as Householder matrices. They are used in numerical methods, e.g. for solving systems of linear equations, least square problems, Eigenvalue computations, $Q R$ decomposition, and for transforming symmetric to tridiagonal matrices. [9]
} 

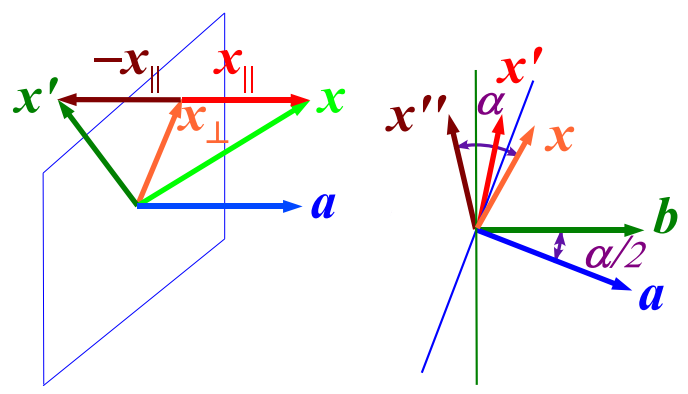

FIGURE 1. Left: Reflection at a hyperplane normal to $\boldsymbol{a}$. Right: Rotation generated by two successive reflections at hyperplanes normal to $\boldsymbol{a}, \boldsymbol{b}$ by twice the angle $\angle(\boldsymbol{a}, \boldsymbol{b})$.

where $\doteq$ means equality up to non-zero scalar factors (which cancel out in (6)). In general the geometric product of $k$ normal vectors (the versor $S$ ) corresponds to the composition of reflections to all symmetry transformations [13] of two-dimensional (2D) and 3D crystal cell point groups (also called crystal classes)

$$
\boldsymbol{x}^{\prime}=(-1)^{k} S^{-1} \boldsymbol{x} S \text {. }
$$

\section{Two dimensional point groups}

2D point groups [13] are generated by multiplying vectors selected [19-21] as in Fig. 2. The index $p$ can be used to denote these groups as in Table 1. For example the hexagonal point group (the symmetry group of the hexagon, leaving the center point invariant) is given by multiplying its two generating vectors $\boldsymbol{a}, \boldsymbol{b}$

$$
6=\left\{\boldsymbol{a}, \boldsymbol{b}, R=\boldsymbol{a} \boldsymbol{b}, R^{2}, R^{3}, R^{4}, R^{5}, R^{6}=-1, \boldsymbol{a} R^{2}, \boldsymbol{b} R^{2}, \boldsymbol{a} R^{4}, \boldsymbol{b} R^{4}\right\} .
$$

In (7) the vectors $\boldsymbol{a}, \boldsymbol{b}$ represent reflections (3) at lines normal to $\boldsymbol{a}, \boldsymbol{b}$ and passing through the center of the hexagon of Fig. 2. The rotor $R=\boldsymbol{a} \boldsymbol{b}$ represents according to (4) a double reflection at the two lines passing through the center of the hexagon of Fig. 2 and normal to $\boldsymbol{a}$ and $\boldsymbol{b}$, respectively. Because $\angle(\boldsymbol{a}, \boldsymbol{b})=30^{\circ}$, the resulting rotation is by $2 \times 30^{\circ}=60^{\circ}$ around the center of the hexagon. The cyclic rotation subgroups are denoted in Table 1 with bars, e.g.

$$
\overline{6}=\left\{R=\boldsymbol{a b}, R^{2}, R^{3}, R^{4}, R^{5}, R^{6}=-1 \doteq 1\right\}
$$

containing the six symmetry rotations of the hexagon of Fig. 2 around its invariant center by $60^{\circ}$, and the multiples $120^{\circ}, 180^{\circ}, 240^{\circ}, 300^{\circ}$, and $360^{\circ}$. The vectors $\boldsymbol{a} R^{2}, \boldsymbol{b} R^{2}, \boldsymbol{a} R^{4}, \boldsymbol{b} R^{4}$ in (7) are the normal directions of the remaining four lines (passing through the center of the hexagon) of reflection symmetry of the hexagon. For further fully interactive illustrations see e.g. the well-commented 2D point group Java applets online available at [16], which include all 2D point groups of Table 1. 
b
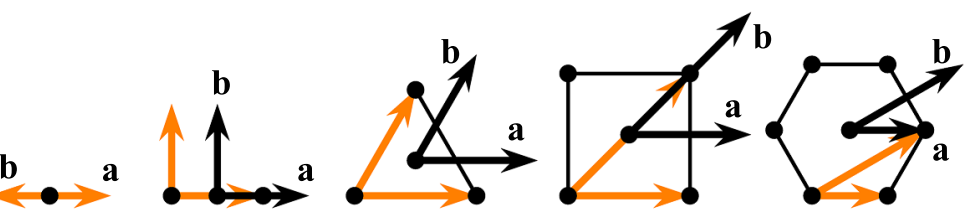

FIGURE 2. Regular polygons $(p=1,2,3,4,6)$ and point group generating vectors $\boldsymbol{a}, \boldsymbol{b}$ subtending angles $\pi / p$ shifted to center.

TABLE 1. Geometric and international notation for 2D point groups.

\begin{tabular}{|c|c|c|c|c|c|}
\hline Crystal & Oblique & Rectangular & Trigonal & Square & Hexagonal \\
\hline geometric & $\overline{1}$ & 1 & 3 & 4 & 6 \\
\hline international & 12 & $\mathrm{~m} \mathrm{~mm}$ & $3 \mathrm{~m} \quad 3$ & $4 \mathrm{~m} \quad 4$ & $6 \mathrm{~m} \quad 6$ \\
\hline
\end{tabular}

\section{Three dimensional point groups}

The selection of three vectors $\boldsymbol{a}, \boldsymbol{b}, \boldsymbol{c}$ from each crystal cell [13,19-21] for generating all 3D point groups is indicated in Fig. 4. The choice of vectors $\boldsymbol{a}, \boldsymbol{b}$ for point groups of tetragonal, trigonal, and hexagonal point groups are further illustrated in Figs. 8, and Figs. 11 to 13 ; for these point groups the third vector $\boldsymbol{c}$ is perpendicular to the $\boldsymbol{a}, \boldsymbol{b}$ plane, such that $\boldsymbol{a}, \boldsymbol{b}, \boldsymbol{c}$ (in this order) form a right handed set of vectors. Finally, the choice of vectors $\boldsymbol{a}, \boldsymbol{b}, \boldsymbol{c}$ for cubic point groups is illustrated in Figs. 14 and 15.

For the purpose of point groups keeping a single cell as a whole invariant, the vectors $\boldsymbol{a}, \boldsymbol{b}, \boldsymbol{c}$ have always to be attached to the invariant center of the respective cell. These three vectors are normals of characteristic hyperplanes passing through the center of the respective crystal cell. The hyperplane reflections which the vectors represent and their combinations as in (3) to (6) constitute all point symmetries of the $3 \mathrm{D}$ crystal cells. The point symmetry transformations are applied to every vertex of a cell and keep the cell as a whole invariant, transforming each vertex into another vertex.

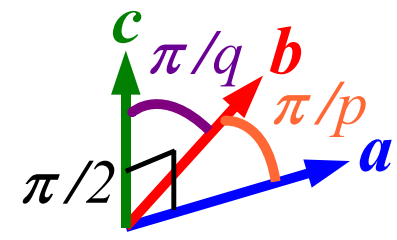

FIGURE 3. Angular relations of pairs of geometric vectors of $\boldsymbol{a}, \boldsymbol{b}, \boldsymbol{c}$ : $\angle(\boldsymbol{a}, \boldsymbol{b})=\pi / p, \angle(\boldsymbol{b}, \boldsymbol{c})=\pi / q, \angle(\boldsymbol{a}, \boldsymbol{c})=\pi / 2, p, q \in\{1,2,3,4,6\}$.

Using $\angle(\boldsymbol{a}, \boldsymbol{b})$ and $\angle(\boldsymbol{b}, \boldsymbol{c})$ (see Fig. 3) we can denote all 32 3D point groups as in Table 2. Again the overbar notation, like e.g. $\bar{p}$ means that the two vectors concerned are only to be used in the fixed rotor combination $\boldsymbol{a b}$. If the closed overbar extends over both 
TABLE 2. Geometric 3D point group symbols [13] and generators with $\boldsymbol{a}, \boldsymbol{b}, \boldsymbol{c}: \angle(\boldsymbol{a}, \boldsymbol{b})=\pi / p, \angle(\boldsymbol{b}, \boldsymbol{c})=\pi / q, \angle(\boldsymbol{a}, \boldsymbol{c})=\pi / 2, p, q \in$ $\{1,2,3,4,6\}$.

\begin{tabular}{lccccccccc}
\hline Symbol & $p=1$ & $p$ & $\bar{p}=\overline{1}$ & $\bar{p}$ & $p q$ & $\bar{p} q$ & $p \bar{q}$ & $\bar{p} \bar{q}$ & $\overline{p q}$ \\
\hline Generators & $\boldsymbol{a}$ & $\boldsymbol{a}, \boldsymbol{b}$ & 1 & $\boldsymbol{a b}$ & $\boldsymbol{a}, \boldsymbol{b}, \boldsymbol{c}$ & $\boldsymbol{a b}, \boldsymbol{c}$ & $\boldsymbol{a}, \boldsymbol{b c}$ & $\boldsymbol{a b}, \boldsymbol{b c}$ & $\boldsymbol{a b c}$ \\
\hline
\end{tabular}

indexes $\overline{p q}$ all three vectors are only to be used in the fixed rotoinversion (alias rotary reflection) combination $\boldsymbol{a b c}$ of (5). For example the monoclinic point groups are then (int. symbols: $2 / \mathrm{m}, \mathrm{m}$ and 2 , respectively)

$$
2 \overline{2}=\{\boldsymbol{c}, R=\boldsymbol{a} \wedge \boldsymbol{b}=i \boldsymbol{c}, i=c R, 1\}, \quad 1=\{\boldsymbol{c}, 1\}, \quad \overline{2}=\{i \boldsymbol{c}, 1\} .
$$

The maximum order point group of the monoclinic cell (called holohedry) has the geometric name $2 \overline{2}$. Looking at the group elements listed in (9), the groups 1 and $\overline{2}$ are obvious subgroups of $2 \overline{2}$. To generate $2 \overline{2}$ we only need two versors: $c$ (therefore 2 in the group name $2 \overline{2}$ ) and $R=\boldsymbol{a} \wedge \boldsymbol{b}=i \boldsymbol{c}$ (therefore $\overline{2}$ in the group name $2 \overline{2}$ ) ${ }^{2}$. Let us point out, that the symbolic notation of Table 2 is fully isomorphic to the Coxeter notation used in Table 2 of [5], where the two indexes $p, q$ play the very same roles.
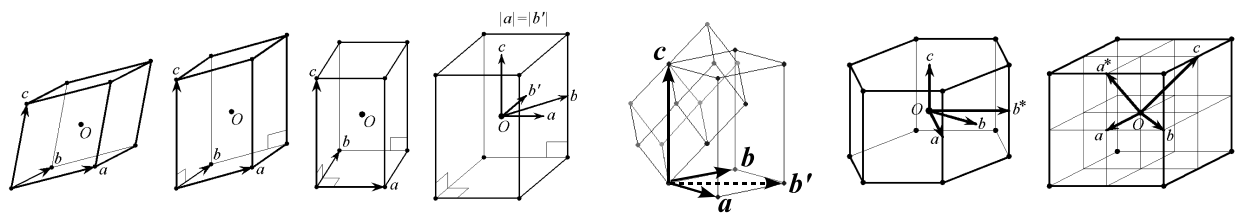

FIGURE 4. 7 crystal cells with vector generators $\boldsymbol{a}, \boldsymbol{b}, \boldsymbol{c}$ : triclinic, monoclinic, orthorhombic, tetragonal, trigonal (rhombohedral), hexagonal, cubic.

\section{Space groups}

The smooth composition with translations is best done in the conformal model $[1,8,15$, $17,18,27]$ of Euclidean space (in the GA of $\mathbb{R}^{4,1}$ ), which adds two null-vector dimensions for the origin $\boldsymbol{e}_{0}$ and infinity $\boldsymbol{e}_{\infty}$

$$
X=\boldsymbol{x}+\frac{1}{2} \boldsymbol{x}^{2} \boldsymbol{e}_{\infty}+\boldsymbol{e}_{0}, \quad \boldsymbol{e}_{0}^{2}=\boldsymbol{e}_{\infty}^{2}=X^{2}=0, \quad X \cdot \boldsymbol{e}_{\infty}=-1
$$

\footnotetext{
${ }^{2}$ The systematic geometric name of the monoclinic holohedry with generators $\boldsymbol{a} \wedge \boldsymbol{b}$ and $\boldsymbol{c}$ should actually be $\overline{2} 2$ (and not $2 \overline{2}$ ). This $\overline{2} 2$ corresponds $1: 1$ to the choice of UNIQUE AXIS $c$ in Table 5 . Applying the systematic construction of generators to the geometric point group symbol $2 \overline{2}$ would instead lead to the versors $\boldsymbol{a}$ and $\boldsymbol{b} \wedge \boldsymbol{c}$. In [14] therefore the following cyclic renaming of vectors $\boldsymbol{a} \rightarrow \boldsymbol{c}, \boldsymbol{b} \rightarrow \boldsymbol{a}$, and $\boldsymbol{c} \rightarrow \boldsymbol{b}$ must be assumed in order to arrive at the versor generators $\boldsymbol{c}$ and $\boldsymbol{a} \wedge \boldsymbol{b}$ from $2 \overline{2}$. Special for monoclinic groups is that in order to correctly represent a $180^{\circ}$ rotation the bivector $\boldsymbol{a} \wedge \boldsymbol{b}$ is chosen instead of the geometric product $\boldsymbol{a} \boldsymbol{b}=\boldsymbol{a} \cdot \boldsymbol{b}+\boldsymbol{a} \wedge \boldsymbol{b}$.
} 
TABLE 3. Computing with reflections and translations. The vectors $\boldsymbol{a}, \boldsymbol{b}$ are pictured in Fig. 2.

\begin{tabular}{cccccc}
\hline$\angle(\boldsymbol{a}, \boldsymbol{b})$ & $180^{\circ}$ & $90^{\circ}$ & $60^{\circ}$ & $45^{\circ}$ & $30^{\circ}$ \\
\hline$T_{\boldsymbol{a}} \boldsymbol{b}=$ & $\boldsymbol{b} T_{-\boldsymbol{a}}$ & $\boldsymbol{b} T_{\boldsymbol{a}}$ & $\boldsymbol{b} T_{\boldsymbol{a}-\boldsymbol{b}}$ & $\boldsymbol{b} T_{\boldsymbol{a}-\boldsymbol{b}}$ & $\boldsymbol{b} T_{\boldsymbol{a}-\boldsymbol{b}}$ \\
$T_{\boldsymbol{b}} \boldsymbol{a}=$ & $\boldsymbol{a} T_{-\boldsymbol{b}}$ & $\boldsymbol{a} T_{\boldsymbol{b}}$ & $\boldsymbol{a} T_{\boldsymbol{b}-\boldsymbol{a}}$ & $\boldsymbol{a} T_{\boldsymbol{b}-2 \boldsymbol{a}}$ & $\boldsymbol{a} T_{\boldsymbol{b}-3 \boldsymbol{a}}$ \\
\hline
\end{tabular}

The inner product of two conformal points gives their Euclidean distance and therefore a plane $m$ equidistant from two points $A, B$ as

$$
X \cdot A=-\frac{1}{2}(\boldsymbol{x}-\boldsymbol{a})^{2} \Rightarrow X \cdot(A-B)=0, \quad m=A-B \propto \boldsymbol{n}-d \boldsymbol{e}_{\infty},
$$

where $\boldsymbol{n}$ is a unit normal to the plane and $d$ its signed scalar distance from the origin. Reflecting at two parallel planes $m, m^{\prime}$ with distance $t / 2$ we get the translation operator (by $t$ )

$$
X^{\prime}=m^{\prime} m X m m^{\prime}=T_{t}^{-1} X T_{t}, \quad T_{t}=1+\frac{1}{2} t e_{\infty} .
$$

Reflection at two non-parallel planes $m, m^{\prime}$ yields the rotation around the $m, m^{\prime}$-intersection by twice the angle subtended by $m, m^{\prime}$.

Group theoretically the conformal group $C(3)$ is isomorphic to $O(4,1)$ and the Euclidean group $E(3)$ is the subgroup of $O(4,1)$ leaving infinity $\boldsymbol{e}_{\infty}$ invariant [13]. Now general translations and rotations are represented by geometric products of vectors (Lipschitz elements, called versors in [13]). To study combinations of versors it is useful to know that (cf. Table 3)

$$
T_{\boldsymbol{t}} \boldsymbol{a}=\boldsymbol{a} T_{\boldsymbol{t}^{\prime}}, \quad \boldsymbol{t}^{\prime}=-\boldsymbol{a}^{-1} \boldsymbol{t} \boldsymbol{a} .
$$

Applying these techniques one can compactly tabulate geometric space group symbols and generators [13]. Table 4 implements this for the 13 monoclinic space groups.

In order to denote Bravais lattices we use the letters $P$ (primitive or principal, one vertex per cell), $I$ (body-centered), $A, B, C$ (single face-centered), $F$ (face-centered), $H$ (hexagonally centered), $R$ (rhombohedral). Primitive lattice cells are illustrated in Figs. 4 , $6,14,15$, and 16, placing one general element at each vertex. Body-centered lattice cells are illustrated in Figs. 9, and 10. Face-centered lattice cells are illustrated in Figs. 7, 20, and 21. All non-primitive Bravais lattices have extra fractional translations as generators, see Fig. 5 (compare also Fig. 6 of [14]).

All this is interactively visualized by the Space Group Visualizer [26]. Fig. 6 shows the SGV screenshot of space group $P 2 \overline{2}_{1}$ (Int. name $P 2_{1} / m$, No. 11 ).

\section{Relations of cell vectors and origins of GA space group generators with conventional crystallographic cells}

In this section we compare crystal cells defined in the course of creating the new crystallographic space group representation in geometric algebra [14] with the conventional crystal cells of the International Tables for Crystallography [11] (referred to as ITA2005). 

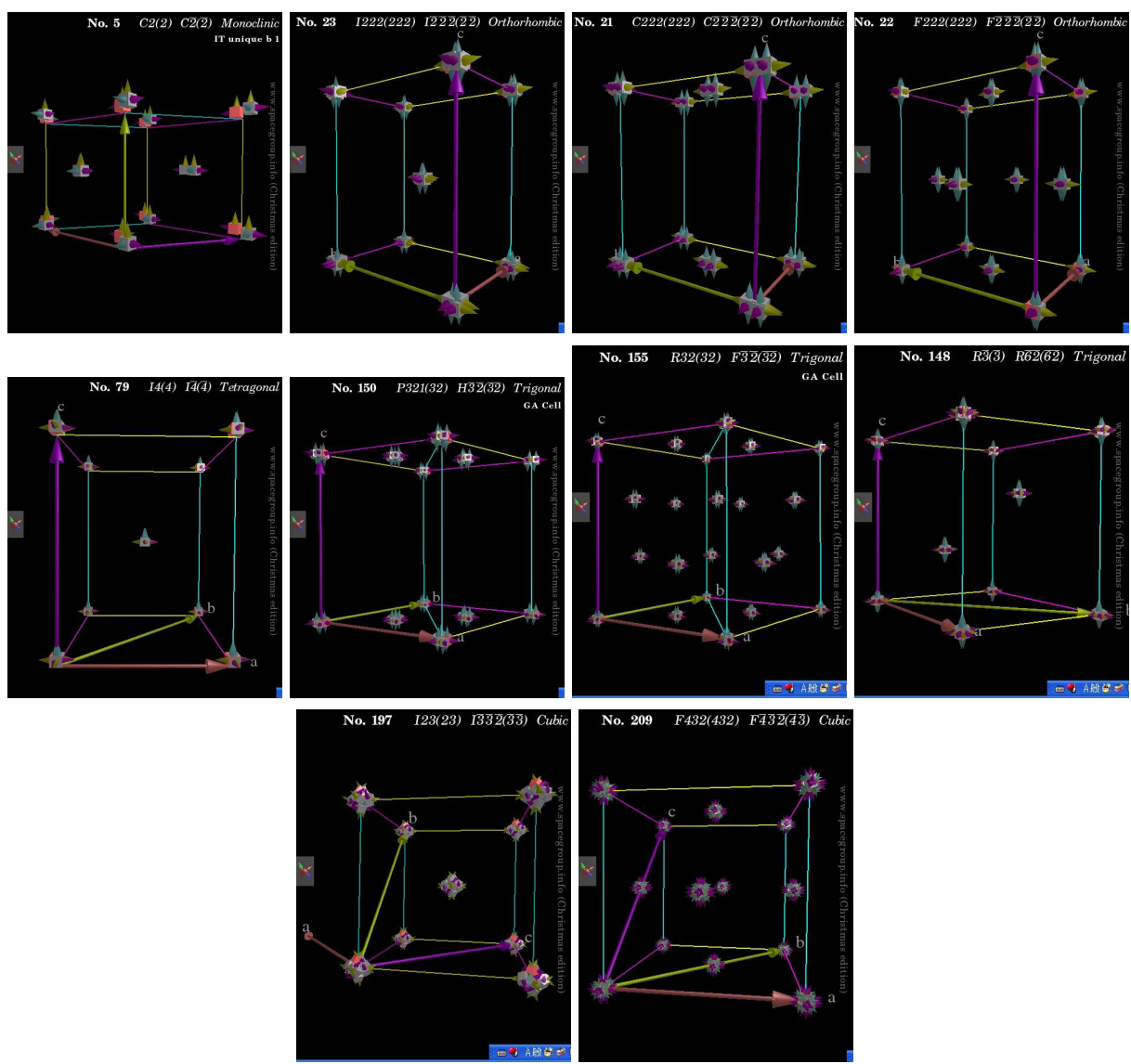

FIGURE 5. Non-primitive Bravais lattices as seen in the SGV. Top row, left to right: Monoclinic $C$ (generator $T^{C}=T_{\boldsymbol{a}+\boldsymbol{b}}^{1 / 2}$ ), orthorhombic $I, C$, and $F$. Center row, left to right: Tetragonal $I$, trigonal/hexagonal $H, F$, and $R$. Bottom row, left to right: Cubic $I$ and $F$.

The definitions of the three-dimensional Bravais lattices and their symmetry vectors in Fig. 5 (cf. also Fig. 6 of [14]) and the explicit GA versor generators of space groups specified in Table 5 of [14] are used with minor modifications in the Space Group Visualizer (SGV) [26]. In [14] the origins of crystal cells and their symmetry are implicitly determined by the generators of Table 5. The SGV makes this explicit in the form of the geometric algebra cell (GA cell).

Yet in crystallography certain centers of symmetry (e.g. centers of inversion symmetry, or intersection points of rotation and screw axis) have deliberately been chosen as points of origin for conventional crystal cells. These conventional cell origins $\mathbf{O}_{\text {ITA2005 }}$ do 


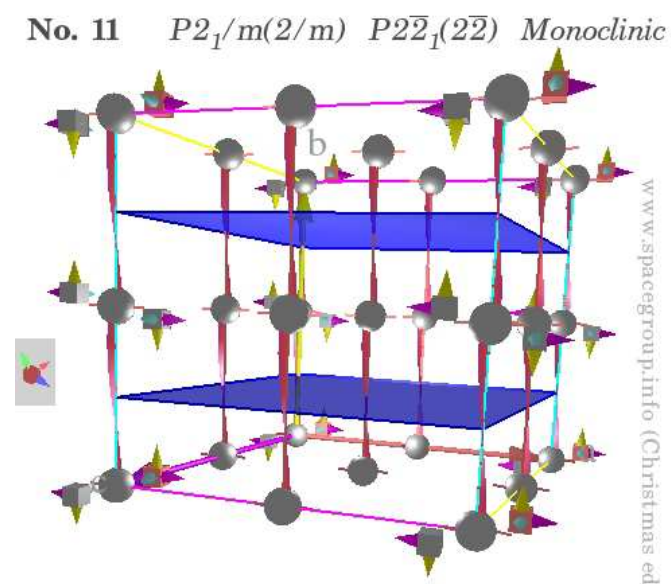

FIGURE 6. SGV screenshot of space group $P 2 \overline{2}_{1}$ (Int. name $P 2_{1} / m$, No. 11), cell choice: UNIQUE AXIS $b$ (see Tables 4 and 5). Inversion centers (gray balls): $\boldsymbol{c} \wedge \boldsymbol{a} \boldsymbol{b} T(k \boldsymbol{a}+\boldsymbol{l} \boldsymbol{b}+m \boldsymbol{c}), k, l, m \in\{0,1,2\}$, screw axis (red): $\boldsymbol{c} \wedge \boldsymbol{a} T\left(k \boldsymbol{a}+\frac{1}{2} \boldsymbol{b}+m \boldsymbol{c}\right), k, m \in\{0,1,2\}$, reflection planes (blue): $\boldsymbol{b} T\left(\left[\frac{1}{2}+l\right] \boldsymbol{b}\right), l \in\{0,1\}$.

TABLE 4. Monoclinic space group versor (Lipschitz element) generators, $T^{A}=T_{\boldsymbol{b}+\boldsymbol{c}}^{1 / 2}$, int. = international [11], geo. = geometric, alt. = alternative, columns 3 and 4: [13]. $T_{\boldsymbol{a}}, T_{\boldsymbol{b}}, T_{\boldsymbol{c}}$ suppressed.

\begin{tabular}{|c|c|c|c|c|c|}
\hline Int.\# & Int. name & Geo. name & Geo. generators & Int. generators & Alt. generators \\
\hline 3 & $P 2$ & $P \overline{2}$ & $i c=a \wedge b$ & & \\
\hline 4 & $P 2_{1}$ & $P \overline{2}_{1}$ & $i c T_{c}^{1 / 2}$ & & \\
\hline 5 & $C 2$ & $A \overline{2}$ & $i c, T^{A}$ & & \\
\hline 6 & $P m$ & $P 1$ & $c$ & & \\
\hline 7 & $P c$ & $P_{a} 1$ & $c T_{a}^{1 / 2}$ & & \\
\hline 8 & $\mathrm{Cm}$ & $A 1$ & $\boldsymbol{c}, T^{A}$ & & \\
\hline 9 & $C c$ & $A_{a} 1$ & $\boldsymbol{c} T_{\boldsymbol{a}}^{1 / 2}, T^{A}$ & & \\
\hline 10 & $P 2 / m$ & $P 2 \overline{2}$ & $c, i c$ & $i, i c$ & $i, c$ \\
\hline 11 & $P 2_{1} / m$ & $P 2 \overline{2}_{1}$ & $\boldsymbol{c}, i c T_{\boldsymbol{c}}^{1 / 2}$ & $i, i c T_{c}^{1 / 2}$ & $i, \boldsymbol{c} T_{\boldsymbol{c}}^{1 / 2}$ \\
\hline 12 & $C 2 / m$ & $A 2 \overline{2}$ & $\boldsymbol{c}, i \boldsymbol{c}, T^{A}$ & $i T^{A}, i c T^{A}, T^{A}$ & $i, \boldsymbol{c}, T^{A}$ \\
\hline 13 & $P 2 / c$ & $P_{a} 2 \overline{2}$ & $\boldsymbol{c} T_{\boldsymbol{a}}^{1 / 2}, i \boldsymbol{c}$ & $i, i c T_{\boldsymbol{a}}^{1 / 2}$ & $i, c T_{a}^{1 / 2}$ \\
\hline 14 & $P 2_{1} / c$ & $P_{a} 2 \overline{2}_{1}$ & $\boldsymbol{c} T_{\boldsymbol{a}}^{1 / 2}, i \boldsymbol{c} T_{\boldsymbol{c}}^{1 / 2}$ & $i, i c T_{\boldsymbol{a}+\boldsymbol{c}}^{1 / 2}$ & $i, \boldsymbol{c} T_{\boldsymbol{a}+\boldsymbol{c}}^{1 / 2}$ \\
\hline 15 & $C 2 / c$ & $A_{a} 2 \overline{2}$ & $\boldsymbol{c} T_{\boldsymbol{a}}^{1 / 2}, i c, T^{A}$ & $i, i c T_{\boldsymbol{a}}^{1 / 2}, T^{A}$ & $i, c T_{\boldsymbol{a}}^{1 / 2}, T^{A}$ \\
\hline
\end{tabular}


in many cases differ from the GA Cell origins $\mathbf{O}_{\mathrm{GA}}$ Cell. Similarly the Bravais lattices defined in Fig. 5 (cf. also Fig. 6 of [14]) and in sections 2.1.3 and 9.1 of [11] are not always identical. We denote the symmetry vectors of [14] used in the SGV with italic lower case bold fonts $\boldsymbol{a}, \boldsymbol{b}$ and $\boldsymbol{c}$. We denote the conventional crystal cell vectors of [11] by upright lower case bold fonts $\mathbf{a}, \mathbf{b}$ and $\mathbf{c}$. We always specify origin shift vectors $\Delta \mathbf{O}$ in the $\{\boldsymbol{a}, \boldsymbol{b}, \boldsymbol{c}\}$ system of geometric symmetry vectors.

We define

$$
\Delta \mathbf{O}_{\mathrm{ITA} 2005}=\mathbf{O}_{\mathrm{ITA} 2005}-\mathbf{O}_{\mathrm{GA} \text { Cell }},
$$

or, if no GA Cell is explicitly declared as such in the SGV, as

$$
\Delta \mathbf{O}_{\mathrm{ITA} 2005}=\mathbf{O}_{\mathrm{ITA} 2005}-\mathbf{O}_{\mathrm{SGV} \text { standard }} \text {. }
$$

In the SGV the notation ITA2005 is not used. Instead cells named IT Cell, IT Cell 1 and IT Cell 2 can be selected in the SGV, and if different the cell named GA Cell. The position, size, shape and orientation of a cell depicted in a space group entry in [11] corresponds to the IT Cell. If [11] provides several diagrams entitled CELL CHOICE 1 (or ORIGIN CHOICE 1) and CELL CHOICE 2 (or ORIGIN CHOICE 2), then these will corresponds in position, size, shape and orientation to IT Cell 1 and IT Cell 2, respectively. This leads to the definitions

$$
\begin{aligned}
\Delta \mathbf{O}_{\text {IT Cell }} & =\mathbf{O}_{\text {IT Cell }}-\mathbf{O}_{\mathrm{GA} \mathrm{Cell}}, \\
\Delta \mathbf{O}_{\mathrm{IT} \mathrm{Cell} \mathrm{1}} & =\mathbf{O}_{\text {IT Cell 1 }}-\mathbf{O}_{\mathrm{GA} \mathrm{Cell}}, \\
\Delta \mathbf{O}_{\mathrm{IT} \mathrm{Cell} \mathrm{2}} & =\mathbf{O}_{\mathrm{IT} \mathrm{Cell} \mathrm{2}}-\mathbf{O}_{\mathrm{GA} \mathrm{Cell}} .
\end{aligned}
$$

In the following we conduct the comparison for each crystal family. Where necessary additional crystal family specific information is given, including family specific notation. For ease of notation we refer to space groups by numbers from $\mathbf{1}$ to $\mathbf{2 3 0}$ as used in [11] and [14].

\subsection{Triclinic space groups \# 1,2}

In this case both symmetry vectors and origins agree

$$
(\mathbf{a}, \mathbf{b}, \mathbf{c})=(\boldsymbol{a}, \boldsymbol{b}, \boldsymbol{c}), \quad \Delta \mathbf{O}_{\mathrm{ITA} 2005}=0 .
$$

Let us emphasize that we mean with $(\mathbf{a}, \mathbf{b}, \mathbf{c})=(\boldsymbol{a}, \boldsymbol{b}, \boldsymbol{c})$ a vector by vector identity: $\mathbf{a}=\boldsymbol{a}$, $\mathbf{b}=\boldsymbol{b}$, and $\mathbf{c}=\boldsymbol{c}$.

\subsection{Monoclinic space groups \# 3-15}

For monoclinic cells the origins are always identical

$$
\Delta \mathbf{O}_{\mathrm{ITA} 2005}=0
$$

and the symmetry vectors of [14] agree with the cells referred to in ITA2005 as UNIQUE AXIS $b$ or UNIQUE AXIS $b$, CELL CHOICE 1 . The details are given in Table 5 . 
TABLE 5. Comparison of geometric symmetry vectors [14] and conventional cell vectors (ITA2005) for monoclinic cells.

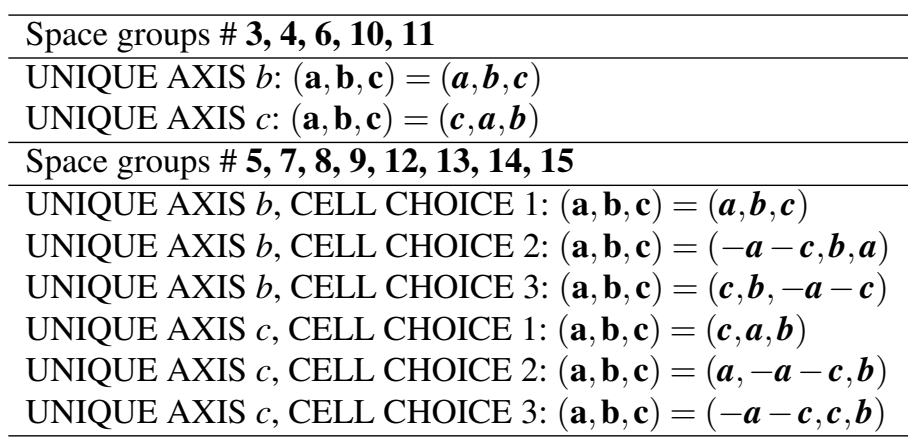

\subsection{Orthorhombic space groups \# 16-74}

The key reference figure apart from the individual space group entries in ITA2005 is Fig. 2.2.6.5. of ITA2005. Note well that the symmetry vectors of [14] and the conventional cell vectors of ITA2005 agree always

$$
(\mathbf{a}, \mathbf{b}, \mathbf{c})=(\boldsymbol{a}, \boldsymbol{b}, \boldsymbol{c})
$$

except for space group \# $\mathbf{1 8}$ for which we currently have in the SGV

$$
(\mathbf{a}, \mathbf{b}, \mathbf{c})=(\boldsymbol{c}, \boldsymbol{a}, \boldsymbol{b}) .
$$

Table 6 therefore lists only the origin shifts. The alternative origin shifts in the third column of Table 6 lead to fully equivalent cells, but are listed because they are the ones actually used in the SGV. As an example Fig. 7 shows the three views (IT Cell 1, IT Cell 2, GA Cell) of the orthorhombic space group $F_{d} 2_{d} 2_{d}$ (Int. name $F d d d$, No. 70) as generated in the Space Group Visualizer. To avoid cluttering, only the rotation axis are shown. The IT Cell 1 view differs in its choice of origin from the other two views, as denoted in Table 6. Actually the IT Cell 2 and GA Cell also have different but equivalent choices of origin, as can easily be seen by comparing the center and right parts of Fig. 7 .

\subsection{Tetragonal space groups \# 75-142}

The key reference figure apart from the individual space group entries in ITA2005 is Fig. 2.2.6.7. of ITA2005. Note well that the symmetry vectors of [14] and the conventional cell vectors of ITA2005 are always related by (see Fig. 8)

$$
(\mathbf{a}, \mathbf{b}, \mathbf{c})=(\boldsymbol{a}, \boldsymbol{b}-\boldsymbol{a}, \boldsymbol{c}) .
$$

Tables 7 and 8 therefore list only the origin shifts. The alternative origin shifts in the third columns of tables 7 and 8 lead to fully equivalent cells, but are listed because they are the ones actually used in the SGV. Table 8 lists the tetragonal groups which have two origin choices in ITA2005.

As an example Fig. 9 shows the two views (IT Cell, GA Cell) of the tetragonal space group $I \overline{4}_{1} \overline{2} \overline{2}$ (Int. name $I 4_{1} 22$, No. 98 ) as generated in the Space Group Visualizer. 


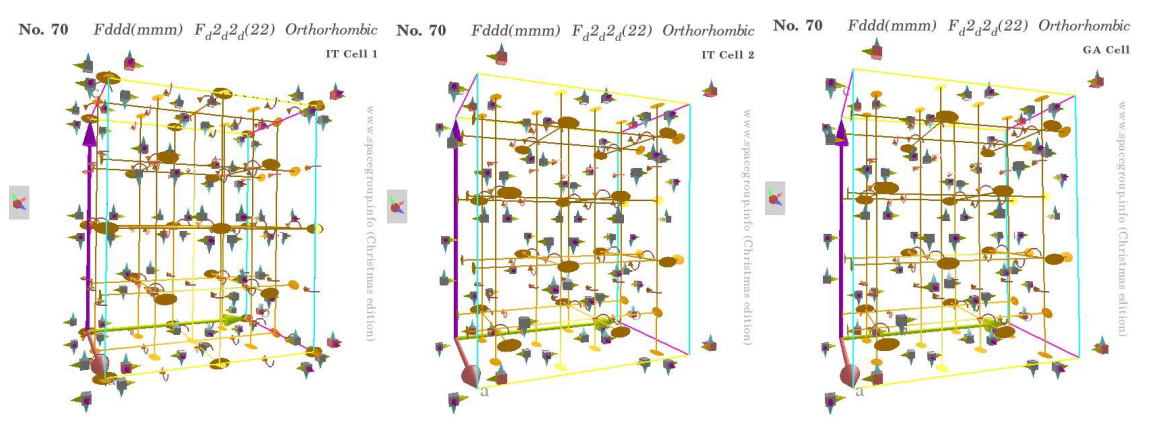

FIGURE 7. SGV views of orthorhombic space group $F_{d} 2{ }_{d} 2_{d}$ (Int. name $F d d d$, No. 70), compare Table 6. Left: IT Cell 1 (ORIGIN CHOICE 1). Center: IT Cell 2 (ORIGIN CHOICE 2). Right: GA Cell.

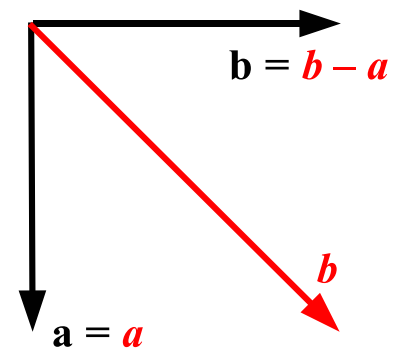

FIGURE 8. Tetragonal cell symmetry vectors $\boldsymbol{a}, \boldsymbol{b}$ of [14] and the conventional cell vectors $\mathbf{a}, \mathbf{b}$ of ITA2005.

To avoid cluttering, only the $90^{\circ}$ screw axis are shown. The IT Cell view differs in its choice of origin from the GA Cell view, which is clearly seen from the different positions of the $90^{\circ}$ screw axis in the $\boldsymbol{a b}$-plane, and denoted in Table 7.

A second example Fig. 10 shows the three views (IT Cell 1, IT Cell 2, GA Cell) of the tetragonal space group $I \overline{4}_{1 a} 2$ (Int. name $I 4_{1} / a$, No. 88) as generated in the Space Group Visualizer. All symmetries located in the cell are shown: inversions (grey balls), glide reflections (gold, $70 \%$ plane size), $90^{\circ}$ screw axis (blue), $90^{\circ}$ rotoinversions (pale red). The three views differ in their origins, as can clearly be seen comparing the level of the glide planes, and of the blue $90^{\circ}$ screw axis, and as denoted in Table 8 .

\subsection{Trigonal space groups \# 143-167}

The key reference figures apart from the individual space group entries in ITA2005 are Fig. 2.2.6.8. and Fig. 2.2.6.9. of ITA2005. For the (obverse) rhombohedral setting Fig. 5.1.3.6.(a) and Fig. 5.1.3.6.(b) of ITA2005 serve as reference. The (obverse) rhombohedral cell vectors $\mathbf{a}_{r}, \mathbf{b}_{r}$, and $\mathbf{c}_{r}$ of ITA2005 [compare also the trigonal (rhombohedral) cell 
TABLE 6. Comparison of geometric symmetry vectors [14] and conventional cell vectors (ITA2005) for orthorhombic cells.

\begin{tabular}{|c|c|c|}
\hline Space group \# & Origin shift & Alternative \\
\hline $\begin{array}{c}16,17,20,21,22,23,25,26 \\
27,31,35,36,37,38,42 \\
44,47,49,65,66,69,71\end{array}$ & $\Delta \mathbf{O}_{\mathrm{ITA} 2005}=0$ & \\
\hline 18 & $\Delta \mathbf{O}_{\text {IT Cell }}=\frac{1}{4} \boldsymbol{a}+\frac{1}{4} \boldsymbol{c}$ & \\
\hline $19,28,29,40,46,51,54,67$ & $\Delta \mathbf{O}_{\mathrm{IT} \mathrm{Cell}}=\frac{1}{4} \boldsymbol{a}$ & $\#$ 67: $\Delta \mathbf{O}_{\text {IT Cell }}=\frac{1}{4} \boldsymbol{b}$ \\
\hline $24,30,39,52$ & $\Delta \mathbf{O}_{\mathrm{IT} \text { Cell }}=\frac{1}{4} \boldsymbol{b}$ & \\
\hline 53,63 & $\Delta \mathbf{O}_{\text {IT Cell }}=\frac{1}{4} \boldsymbol{c}$ & \\
\hline $32,33,34,41,45,55,56,58,72$ & $\Delta \mathbf{O}_{\text {IT Cell }}=\frac{1}{4} \boldsymbol{a}+\frac{1}{4} \boldsymbol{b}$ & \\
\hline 43 & $\Delta \mathbf{O}_{\text {IT Cell }}=\frac{1}{8} \boldsymbol{a}-\frac{1}{8} \boldsymbol{b}$ & $\frac{3}{8} \boldsymbol{a}+\frac{3}{8} \boldsymbol{b}$ \\
\hline $57,74,64$ & $\Delta \mathbf{O}_{\text {IT Cell }}=\frac{1}{4} \boldsymbol{b}+\frac{1}{4} \boldsymbol{c}$ & \\
\hline 60 & $\Delta \mathbf{O}_{\text {IT Cell }}=\frac{1}{4} \boldsymbol{a}+\frac{1}{4} \boldsymbol{c}$ & \\
\hline $61,62,73$ & $\Delta \mathbf{O}_{\text {IT Cell }}=\frac{1}{4} \boldsymbol{a}+\frac{1}{4} \boldsymbol{b}+\frac{1}{4} \boldsymbol{c}$ & \\
\hline 48: ORIGIN CHOICE 1 & $\Delta \mathbf{O}_{\text {IT Cell } 1}=\frac{1}{4} \boldsymbol{a}+\frac{1}{4} \boldsymbol{b}+\frac{1}{4} \boldsymbol{c}$ & \\
\hline 48: ORIGIN CHOICE 2 & $\Delta \mathbf{O}_{\text {IT Cell } 2}=0$ & \\
\hline 50: ORIGIN CHOICE 1 & $\Delta \mathbf{O}_{\text {IT Cell } 1}=\frac{1}{4} \boldsymbol{a}+\frac{1}{4} \boldsymbol{b}$ & \\
\hline 50: ORIGIN CHOICE 2 & $\Delta \mathbf{O}_{\text {IT Cell } 2}=0$ & \\
\hline 59: ORIGIN CHOICE 1 & $\Delta \mathbf{O}_{\mathrm{IT} \mathrm{Cell} 1}=0$ & \\
\hline 59: ORIGIN CHOICE 2 & $\Delta \mathbf{O}_{\text {IT Cell } 2}=\frac{1}{4} \boldsymbol{a}+\frac{1}{4} \boldsymbol{b}$ & \\
\hline 68: ORIGIN CHOICE 1 & $\Delta \mathbf{O}_{\text {IT Cell } 1}=\frac{1}{4} \boldsymbol{a}+\frac{1}{4} \boldsymbol{b}+\frac{1}{4} \boldsymbol{c}$ & \\
\hline 68: ORIGIN CHOICE 2 & $\Delta \mathbf{O}_{\text {IT Cell } 2}=\frac{1}{4} \boldsymbol{a}$ & \\
\hline 70: ORIGIN CHOICE 1 & $\Delta \mathbf{O}_{\text {IT Cell } 1}=-\frac{1}{8} \boldsymbol{a}-\frac{1}{8} \boldsymbol{b}-\frac{1}{8} \boldsymbol{c}$ & $\frac{3}{8} \boldsymbol{a}+\frac{3}{8} \boldsymbol{b}-\frac{1}{8} \boldsymbol{c}$ \\
\hline 70: ORIGIN CHOICE 2 & $\Delta \mathbf{O}_{\text {IT Cell } 2}=0$ & $\frac{1}{2} \boldsymbol{a}+\frac{1}{2} \boldsymbol{b}$ \\
\hline
\end{tabular}

of Fig. 4] are thus related to the hexagonal cell vectors $\mathbf{a}, \mathbf{b}$, and $\mathbf{c}$ of ITA2005 by

$$
\left(\mathbf{a}_{r}, \mathbf{b}_{r}, \mathbf{c}_{r}\right)=\left(\frac{2}{3} \mathbf{a}+\frac{1}{3} \mathbf{b}+\frac{1}{3} \mathbf{c},-\frac{1}{3} \mathbf{a}+\frac{1}{3} \mathbf{b}+\frac{1}{3} \mathbf{c},-\frac{1}{3} \mathbf{a}-\frac{2}{3} \mathbf{b}+\frac{1}{3} \mathbf{c}\right) .
$$

Space group vectors and origins of trigonal crystal cells characterized by the geometric symmetry vectors $\boldsymbol{a}$ and $\boldsymbol{b}$ enclosing the angle $60^{\circ}=180^{\circ} / 3$ as shown in Fig. 11 are compared in Table 9 . The geometric symmetry vectors and origins $\mathbf{O}_{\mathrm{GA}}$ Cell of trigonal space groups with Bravais lattices $F$ or $H$ (Fig. 5, cf. also Fig. 6 of [14]) are shown in Fig. 12 together with the cell vectors and the origins $\mathbf{O}_{\text {ITA2005 }}$ used in ITA2005. Space 

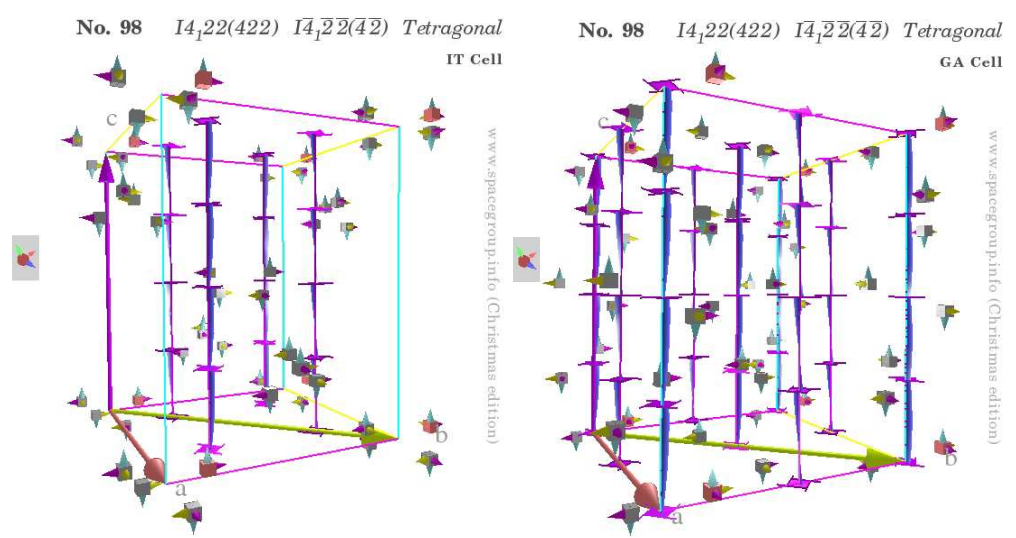

FIGURE 9. SGV views of tetragonal space group $I \overline{4}_{1} \overline{2} \overline{2}$ (Int. name I4 22 , No. 98), compare Table 7. Left: IT Cell. Right: GA Cell.
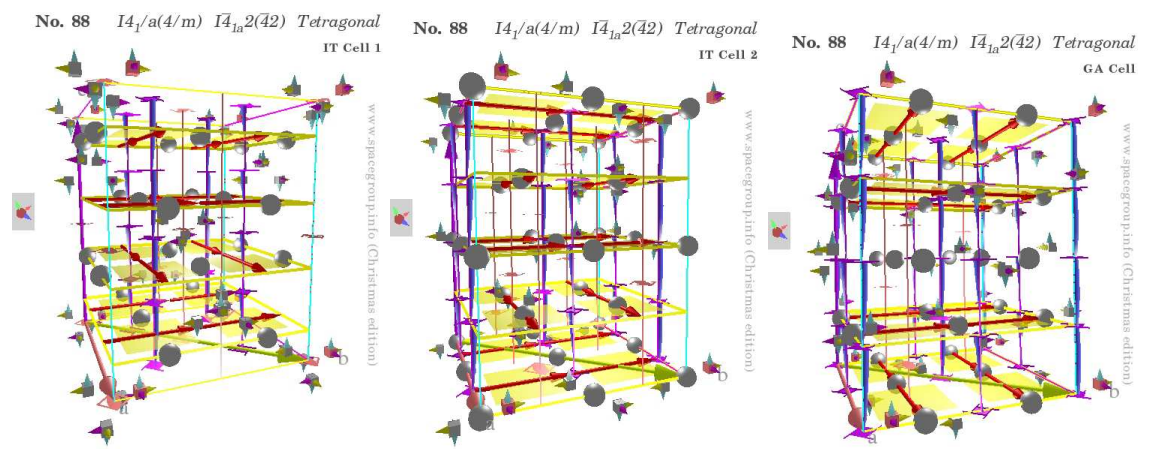

FIGURE 10. SGV views of tetragonal space group $I \overline{4}_{1 a} 2$ (Int. name I4 $1 / a$, No. 88), compare Table 8. Left: IT Cell 1 (ORIGIN CHOICE 1). Center: IT Cell 2 (ORIGIN CHOICE 2). Right: GA Cell.

groups characterized by $\boldsymbol{a}$ and $\boldsymbol{b}$ enclosing the angle $30^{\circ}=180^{\circ} / 6$ as shown in Fig. 13 are compared in Table 10.

For both trigonal and hexagonal space groups the definition of the IT Cell origin shift used in the SGV is

$$
\Delta \mathbf{O}_{\text {IT Cell }}=\Delta \mathbf{O}_{\text {ITA2005 }}+\mathbf{a}
$$

\subsection{Hexagonal space groups \# 168-194}

The key reference figures apart from the individual space group entries in ITA2005 are Fig. 2.2.6.8. and Fig. 2.2.6.9. of ITA2005. 
TABLE 7. Comparison of geometric symmetry vectors [14] and conventional cell vectors (ITA2005) for tetragonal cells with a unique origin in ITA2005.

\begin{tabular}{|c|c|c|}
\hline Space group \# & Origin shift & Alternative \\
\hline $\begin{array}{c}75,76,77,78,79,81,82 \\
83,84,87,89,90,97,99 \\
100,101,103,105,107 \\
111,115,119,121,123 \\
124,131,132,139\end{array}$ & $\Delta \mathbf{O}_{\text {ITA } 2005}=0$ & \\
\hline $104,106,113,117$ & & \\
\hline $127,128,135$ & $\Delta \mathbf{O}_{\text {IT Cell }}=\frac{1}{4} \boldsymbol{b}$ & \\
\hline 91 & $\Delta \mathbf{O}_{\text {IT Cell }}=-\frac{1}{8} \boldsymbol{c}$ & \\
\hline 92,96 & $\Delta \mathbf{O}_{\text {IT Cell }}=\frac{1}{2} \boldsymbol{a}+\frac{1}{4} \boldsymbol{c}$ & \\
\hline $93,112,116,120$ & $\Delta \mathbf{O}_{\text {IT Cell }}=\frac{1}{4} \boldsymbol{c}$ & \\
\hline $94,108,140$ & $\Delta \mathbf{O}_{\text {IT Cell }}=\frac{1}{2} \boldsymbol{a}$ & \\
\hline 95 & $\Delta \mathbf{O}_{\text {IT Cell }}=\frac{1}{8} \boldsymbol{c}$ & \\
\hline $80,98,102,136$ & $\Delta \mathbf{O}_{\text {IT Cell }}=\frac{1}{2} \boldsymbol{a}+\frac{1}{4} \boldsymbol{b}$ & $\begin{array}{l}\# \text { 98: } \frac{1}{2} \boldsymbol{a}-\frac{1}{4} \boldsymbol{b} \\
\# \text { 102, 136: }-\frac{1}{2} \boldsymbol{a}+\frac{1}{4} \boldsymbol{b}\end{array}$ \\
\hline 109 & $\Delta \mathbf{O}_{\text {IT Cell }}=-\frac{1}{4} \boldsymbol{a}+\frac{1}{4} \boldsymbol{b}$ & $-\frac{1}{4} \boldsymbol{a}-\frac{1}{4} \boldsymbol{b}$ \\
\hline 110 & $\Delta \mathbf{O}_{\text {IT Cell }}=\frac{1}{4} \boldsymbol{a}-\frac{1}{4} \boldsymbol{b}$ & \\
\hline 122 & $\Delta \mathbf{O}_{\text {IT Cell }}=\frac{1}{4} \boldsymbol{a}+\frac{1}{8} \boldsymbol{c}$ & $\frac{1}{4} \boldsymbol{a}-\frac{3}{8} \boldsymbol{c}$ \\
\hline 114,118 & $\Delta \mathbf{O}_{\text {IT Cell }}=\frac{1}{4} \boldsymbol{b}+\frac{1}{4} \boldsymbol{c}$ & \\
\hline
\end{tabular}

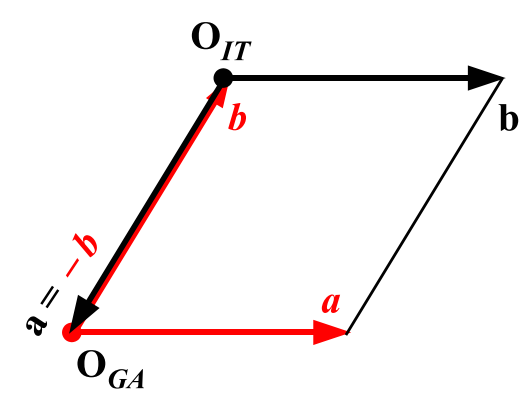

FIGURE 11. Trigonal cell symmetry vectors $\boldsymbol{a}, \boldsymbol{b}$ of [14] enclosing the angle $60^{\circ}=180^{\circ} / 3$, and the conventional cell vectors $\mathbf{a}, \mathbf{b}$ of ITA2005. 
TABLE 8. Comparison of geometric symmetry vectors [14] and conventional cell vectors (ITA2005) for tetragonal cells with two origin choices in ITA2005.

\begin{tabular}{|c|c|c|}
\hline Space group \# & Origin shift & Alternative \\
\hline $85,129,130$ & & \\
\hline ORIGIN CHOICE 1 & $\Delta \mathbf{O}_{\text {IT Cell } 1}=\frac{1}{2} \boldsymbol{a}$ & $\#$ 129, 130: $-\frac{1}{2} \boldsymbol{a}$ \\
\hline ORIGIN CHOICE 2 & $\Delta \mathbf{O}_{\text {IT Cell } 2}=\frac{1}{4} \boldsymbol{b}$ & \\
\hline 86: ORIGIN CHOICE 1 & $\Delta \mathbf{O}_{\text {IT Cell } 1}=\frac{1}{2} \boldsymbol{a}+\frac{1}{4} \boldsymbol{c}$ & \\
\hline 86: ORIGIN CHOICE 2 & $\Delta \mathbf{O}_{\text {IT Cell } 2}=\frac{1}{2} \boldsymbol{a}+\frac{1}{4} \boldsymbol{b}$ & \\
\hline 88: ORIGIN CHOICE 1 & $\Delta \mathbf{O}_{\text {IT Cell } 1}=-\frac{1}{2} \boldsymbol{a}+\frac{1}{4} \boldsymbol{b}+\frac{1}{8} \boldsymbol{c}$ & $\frac{1}{2} \boldsymbol{a}+\frac{1}{4} \boldsymbol{b}+\frac{1}{8} \boldsymbol{c}$ \\
\hline 88: ORIGIN CHOICE 2 & $\Delta \mathbf{O}_{\text {IT Cell } 2}=\frac{1}{4} \boldsymbol{a}+\frac{1}{4} \boldsymbol{c}$ & \\
\hline 125: ORIGIN CHOICE 1 & $\Delta \mathbf{O}_{\text {IT Cell } 1}=\frac{1}{4} \boldsymbol{b}$ & \\
\hline 125: ORIGIN CHOICE 2 & $\Delta \mathbf{O}_{\text {IT Cell 2 }}=0$ & \\
\hline 126: ORIGIN CHOICE 1 & $\Delta \mathbf{O}_{\text {IT Cell } 1}=\frac{1}{4} \boldsymbol{b}+\frac{1}{4} \boldsymbol{c}$ & \\
\hline 126: ORIGIN CHOICE 2 & $\Delta \mathbf{O}_{\text {IT Cell } 2}=0$ & \\
\hline 133,134 & & \\
\hline $\begin{array}{l}\text { ORIGIN CHOICE } 1 \\
\text { ORIGIN CHOICE } 2\end{array}$ & $\begin{aligned} \Delta \mathbf{O}_{\text {IT Cell } 1} & =-\frac{1}{2} \boldsymbol{a}+\frac{1}{4} \boldsymbol{b}+\frac{1}{4} \boldsymbol{c} \\
\Delta \mathbf{O}_{\text {IT Cell } 2} & =0\end{aligned}$ & \\
\hline 137,138 & & \\
\hline ORIGIN CHOICE 1 & $\Delta \mathbf{O}_{\text {IT Cell } 1}=\frac{1}{2} \boldsymbol{a}+\frac{1}{4} \boldsymbol{c}$ & $-\frac{1}{2} \boldsymbol{a}+\frac{1}{4} \boldsymbol{c}$ \\
\hline ORIGIN CHOICE 2 & $\Delta \mathbf{O}_{\text {IT Cell } 2}=\frac{1}{4} \boldsymbol{b}$ & \\
\hline 141: ORIGIN CHOICE 1 & $\Delta \mathbf{O}_{\text {IT Cell } 1}=-\frac{1}{4} \boldsymbol{a}+\frac{1}{4} \boldsymbol{b}+\frac{1}{8} \boldsymbol{c}$ & $\frac{3}{4} \boldsymbol{a}+\frac{1}{4} \boldsymbol{b}+\frac{1}{8} \boldsymbol{c}$ \\
\hline 141: ORIGIN CHOICE 2 & $\Delta \mathbf{O}_{\text {IT Cell } 2}=\frac{1}{4} \boldsymbol{c}$ & \\
\hline 142: ORIGIN CHOICE 1 & $\Delta \mathbf{O}_{\text {IT Cell } 1}=\frac{1}{4} \boldsymbol{a}+\frac{1}{4} \boldsymbol{b}+\frac{1}{8} \boldsymbol{c}$ & \\
\hline 142: ORIGIN CHOICE 2 & $\Delta \mathbf{O}_{\text {IT Cell } 2}=\frac{1}{2} \boldsymbol{a}+\frac{1}{4} \boldsymbol{c}$ & \\
\hline
\end{tabular}

Space group vectors and origins of hexagonal crystal cells characterized by the geometric symmetry vectors $\boldsymbol{a}$ and $\boldsymbol{b}$ enclosing the angle $30^{\circ}=180^{\circ} / 6$ are compared in Table 11 . Note well that for this case the symmetry vectors of [14] and the conventional cell vectors of ITA2005 are always related by

$$
(\mathbf{a}, \mathbf{b}, \mathbf{c})=(\boldsymbol{a}-\boldsymbol{b}, \boldsymbol{a}, \boldsymbol{c}),
$$

a relationship, which is also illustrated in Fig. 13. Space groups characterized by $\boldsymbol{a}$ and $\boldsymbol{b}$ enclosing the angle $60^{\circ}=180^{\circ} / 3$ are compared in Table 12 . The geometric symmetry vectors of [14] and of ITA2005 are illustrated for space groups 174, 187, 188 in Fig. 11, and for space groups 189, 190 in Fig. 12, respectively. 
TABLE 9. Comparison of geometric symmetry vectors [14] and conventional cell vectors (ITA2005) and origin shifts for trigonal cells with $\boldsymbol{a}$ and $\boldsymbol{b}$ enclosing the angle $60^{\circ}=180^{\circ} / 3$.

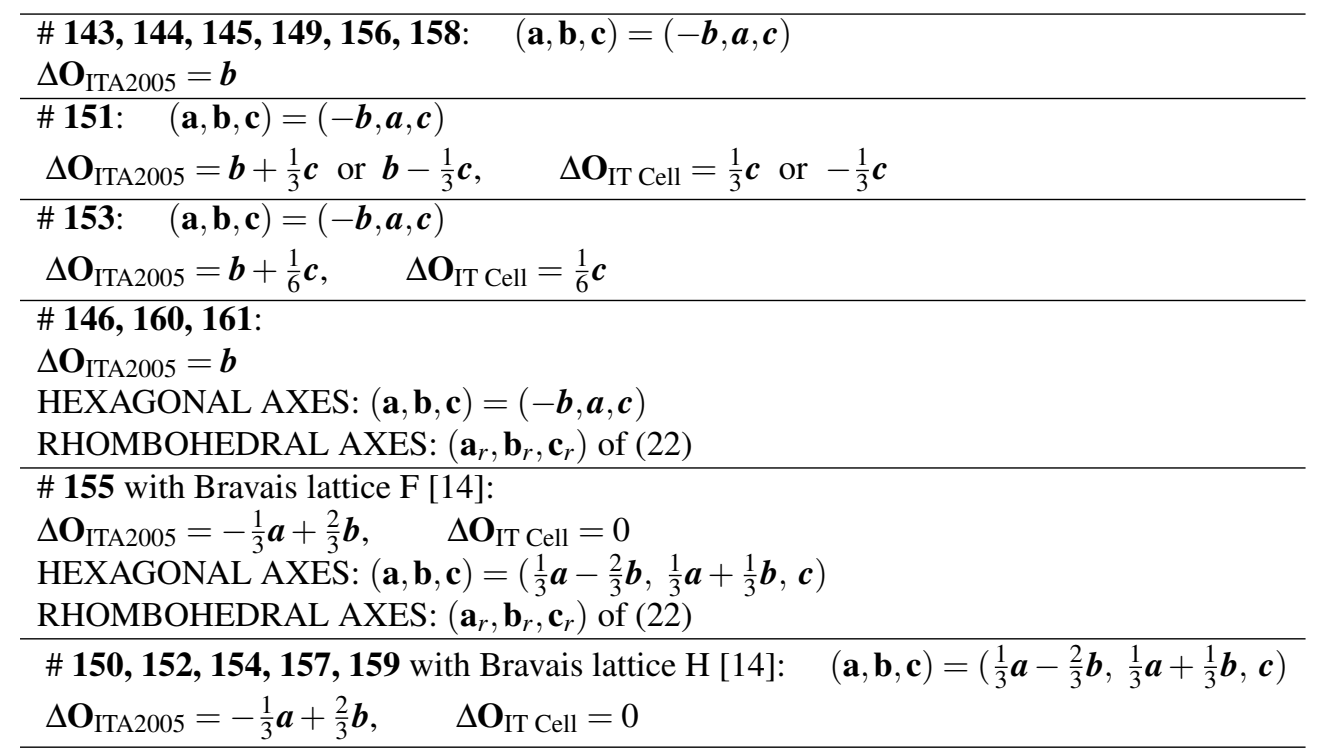

TABLE 10. Comparison of geometric symmetry vectors [14] and conventional cell vectors (ITA2005) and origin shifts for trigonal cells with $\boldsymbol{a}$ and $\boldsymbol{b}$ enclosing the angle $30^{\circ}=180^{\circ} / 6$.

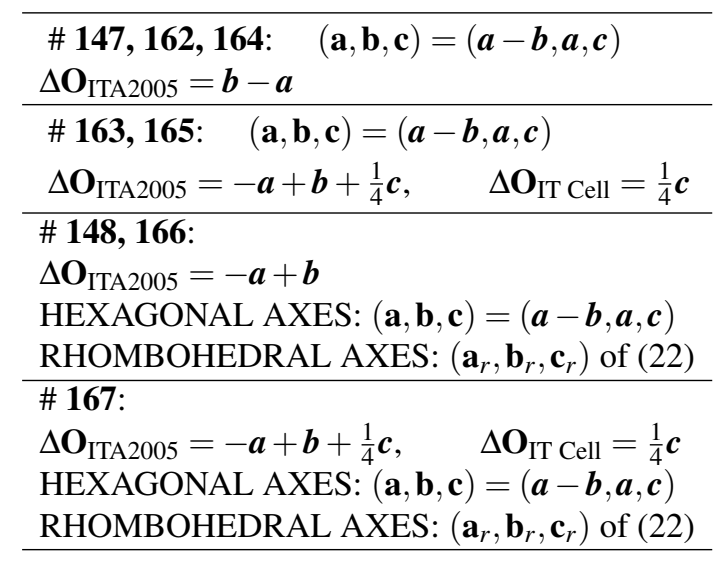




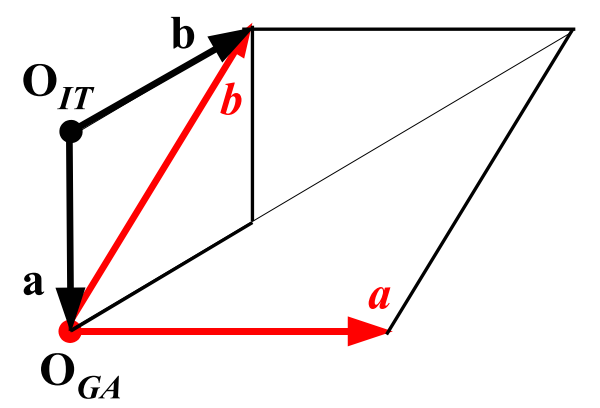

FIGURE 12. Trigonal cell symmetry vectors $\boldsymbol{a}, \boldsymbol{b}$ of [14] enclosing the angle $60^{\circ}=180^{\circ} / 3$, and the conventional cell vectors $\mathbf{a}, \mathbf{b}$ of ITA2005. In the geometric algebra representation of [14] the Bravais lattices $F$ or $H$ are used (Fig. 5, cf. also Fig. 6 of [14]).

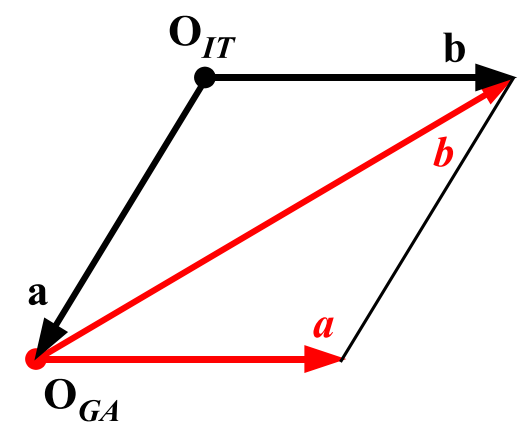

FIGURE 13. Trigonal cell symmetry vectors $\boldsymbol{a}, \boldsymbol{b}$ of [14] enclosing the angle $30^{\circ}=180^{\circ} / 6$, and the conventional cell vectors $\mathbf{a}, \mathbf{b}$ of ITA2005.

\subsection{Cubic space groups \# 195-230}

The key reference figure apart from the individual space group entries in ITA2005 is Fig. 2.2.6.10. of ITA2005.

Space group vectors and origins of cubic crystal cells characterized by the geometric symmetry vectors $\boldsymbol{a}$ and $\boldsymbol{b}$ enclosing the angle $60^{\circ}=180^{\circ} / 3$, and $\boldsymbol{b}$ and $\boldsymbol{c}$ enclosing the same angle, are compared in Table 13 . Note well that for this case the symmetry vectors of [14] and the conventional cell vectors of ITA2005 are always related by (see Fig. 14)

$$
(\mathbf{a}, \mathbf{b}, \mathbf{c})=\left(-\frac{1}{2} \boldsymbol{a}+\frac{1}{2} \boldsymbol{c}, \frac{1}{2} \boldsymbol{a}+\frac{1}{2} \boldsymbol{c},-\frac{1}{2} \boldsymbol{a}+\boldsymbol{b}-\frac{1}{2} \boldsymbol{c}\right) .
$$

Space groups characterized by $\boldsymbol{a}$ and $\boldsymbol{b}$ enclosing the angle $45^{\circ}=180^{\circ} / 4$, and $\boldsymbol{b}$ and $c$ enclosing the angle $60^{\circ}=180^{\circ} / 3$, are compared in Table 14 . Note well that for 
TABLE 11. Comparison of geometric cell origins [14] and conventional cell origins (ITA2005) for hexagonal cells with $\boldsymbol{a}$ and $\boldsymbol{b}$ enclosing the angle $30^{\circ}=180^{\circ} / 6$.

\# 168, 169, 170, 171, 172, 173, 175, 177, 182, 183, 184, 185, 186, 191, 192 :

$\Delta \mathbf{O}_{\text {ITA2005 }}=-\boldsymbol{a}+\boldsymbol{b}$

\# 176, 193, 194:

$\Delta \mathbf{O}_{\text {ITA2005 }}=-\boldsymbol{a}+\boldsymbol{b}+\frac{1}{4} \boldsymbol{c}, \quad \Delta \mathbf{O}_{\text {IT Cell }}=\frac{1}{4} \boldsymbol{c}$

\# 179, 180:

$\Delta \mathbf{O}_{\text {ITA2005 }}=-\boldsymbol{a}+\boldsymbol{b}+\frac{1}{6} \boldsymbol{c}, \quad \Delta \mathbf{O}_{\text {IT Cell }}=\frac{1}{6} \boldsymbol{c}$

\# 178, 181:

$\Delta \mathbf{O}_{\mathrm{ITA} 2005}=-\boldsymbol{a}+\boldsymbol{b}+\frac{1}{3} \boldsymbol{c}$ or $-\boldsymbol{a}+\boldsymbol{b}-\frac{1}{6} \boldsymbol{c}, \quad \Delta \mathbf{O}_{\mathrm{IT} \mathrm{Cell}}=\frac{1}{3} \boldsymbol{c}$ or $-\frac{1}{6} \boldsymbol{c}$

TABLE 12. Comparison of geometric cell origins and symmetry vectors [14] and conventional cell origins and vectors (ITA2005) for hexagonal cells with $\boldsymbol{a}$ and $\boldsymbol{b}$ enclosing the angle $60^{\circ}=180^{\circ} / 3$.
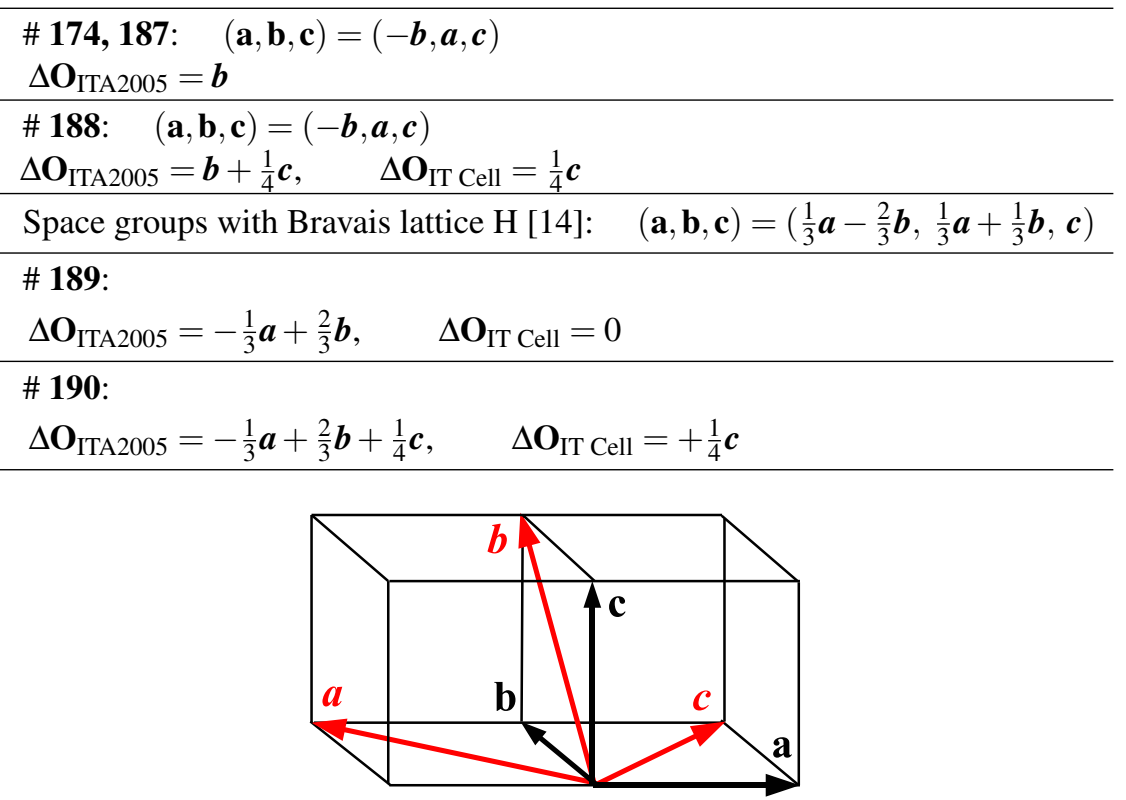

FIGURE 14. Cubic cell symmetry vectors characterized by $\boldsymbol{a}$ and $\boldsymbol{b}$ enclosing the angle $60^{\circ}=180^{\circ} / 3$, and $\boldsymbol{b}$ and $\boldsymbol{c}$ enclosing the same angle, and the conventional cell vectors $\mathbf{a}, \mathbf{b}, \mathbf{c}$ of ITA2005. 


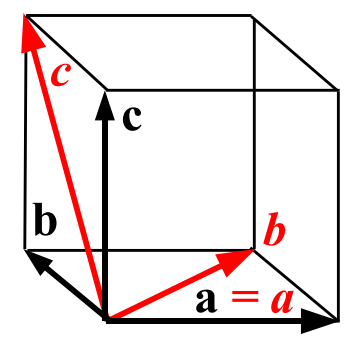

FIGURE 15. Cubic cell symmetry vectors characterized by $\boldsymbol{a}$ and $\boldsymbol{b}$ enclosing the angle $45^{\circ}=180^{\circ} / 4$, and $\boldsymbol{b}$ and $\boldsymbol{c}$ enclosing the angle $60^{\circ}=180^{\circ} / 3$, and the conventional cell vectors $\mathbf{a}, \mathbf{b}, \mathbf{c}$ of ITA2005.

TABLE 13. Comparison of geometric symmetry vectors [14] and conventional cell vectors (ITA2005) for cubic cells with geometric symmetry vectors $\boldsymbol{a}$ and $\boldsymbol{b}$ enclosing the angle $60^{\circ}=180^{\circ} / 3$, and $\boldsymbol{b}$ and $\boldsymbol{c}$ enclosing the same angle.

\begin{tabular}{cll}
\hline Space group \# & Origin shift & Alternative \\
\hline $\mathbf{1 9 5}, \mathbf{1 9 6}, \mathbf{1 9 7}, \mathbf{2 1 5}$ & & \\
$\mathbf{2 1 6 , 2 1 7}, \mathbf{2 1 8}, \mathbf{2 2 0}$ & $\Delta \mathbf{O}_{\text {ITA2005 }}=0$ & \\
\hline $\mathbf{1 9 8}, \mathbf{1 9 9}$ & $\Delta \mathbf{O}_{\text {IT Cell }}=\frac{1}{4} \boldsymbol{a}+\frac{1}{4} \boldsymbol{c}$ & \\
\hline $\mathbf{2 1 9}$ & $\Delta \mathbf{O}_{\text {IT Cell }}=\frac{1}{4} \boldsymbol{b}$ & $-\frac{1}{8} \boldsymbol{a}+\frac{1}{8} \boldsymbol{c}$ \\
\hline
\end{tabular}

this case the symmetry vectors of [14] and the conventional cell vectors of ITA2005 are always related by (see Fig. 15)

$$
(\mathbf{a}, \mathbf{b}, \mathbf{c})=(\boldsymbol{a}, \boldsymbol{b}-\boldsymbol{a}, \boldsymbol{c}+\boldsymbol{a}-\boldsymbol{b}) .
$$

\section{Interactive Software Implementation}

The realization in software relies on the visual multivector software CLUCalc [24, 25]. The excellent graphics rendering is based on OpenGL graphics. The space group symmetry definitions described in the previous sections are denoted for each space group in the form of an XML input file. The XML files serve as input for a CLUCalc script named Space Group Visualizer (SGV) [26].

\subsection{The Space Group Visualizer GUI}

Fig. 16 shows the graphical user interface (GUI) of the SGV. The SGV toolbar is magnified and annotated in Fig. 17. Depending on the displayed space group, basis vector 
TABLE 14. Comparison of geometric symmetry vectors [14] and conventional cell vectors (ITA2005) for cubic cells with geometric symmetry vectors $\boldsymbol{a}$ and $\boldsymbol{b}$ enclosing the angle $45^{\circ}=180^{\circ} / 4$, and $\boldsymbol{b}$ and $\boldsymbol{c}$ enclosing the angle $60^{\circ}=180^{\circ} / 3$.

\begin{tabular}{|c|c|c|}
\hline Space group \# & Origin shift & Alternative \\
\hline \multicolumn{3}{|l|}{$200,202,204,207$} \\
\hline \multicolumn{3}{|l|}{$208,209,210,211$} \\
\hline $212,214,223,225$ & \multicolumn{2}{|l|}{$\Delta \mathbf{O}_{\text {ITA2005 }}=0$} \\
\hline \multicolumn{3}{|l|}{226,229} \\
\hline $205,206,230$ & $\Delta \mathbf{O}_{\text {IT Cell }}=-\frac{1}{4} \boldsymbol{a}+\frac{1}{2} \boldsymbol{b}-\frac{1}{4} \boldsymbol{c}$ & $\#$ 230: $\Delta \mathbf{O}_{\text {IT Cell }}=\frac{3}{4} \boldsymbol{a}+\frac{1}{2} \boldsymbol{b}-\frac{1}{4} \boldsymbol{c}$ \\
\hline 212,213 & $\Delta \mathbf{O}_{\text {IT Cell }}=\frac{1}{2} \boldsymbol{a}$ & \\
\hline \multicolumn{3}{|l|}{$201,222,224$} \\
\hline ORIGIN CHOICE 1 & $\Delta \mathbf{O}_{\text {IT Cell } 1}=-\frac{1}{4} \boldsymbol{a}+\frac{1}{2} \boldsymbol{b}+\frac{1}{4} \boldsymbol{c}$ & $\#$ 224: $\Delta \mathbf{O}_{\text {IT Cell } 1}=\frac{1}{4} \boldsymbol{a}-\frac{1}{2} \boldsymbol{b}-\frac{1}{4} \boldsymbol{c}$ \\
\hline \multirow[t]{2}{*}{ ORIGIN CHOICE 2} & $\Delta \mathbf{O}_{\text {IT Cell } 2}=-\frac{1}{2} \boldsymbol{a}+\frac{1}{2} \boldsymbol{b}$ & $\#$ 222: $\Delta \mathbf{O}_{\text {IT Cell } 2}=\frac{1}{2} \boldsymbol{b}+\frac{1}{2} \boldsymbol{c}$ \\
\hline & & $\# 224: \Delta \mathbf{O}_{\text {IT Cell } 2}=\frac{1}{2} \boldsymbol{a}-\frac{1}{2} \boldsymbol{b}$ \\
\hline \multicolumn{3}{|l|}{203,227} \\
\hline ORIGIN CHOICE 1 & $\Delta \mathbf{O}_{\text {IT Cell } 1}=\frac{5}{8} \boldsymbol{a}-\frac{1}{4} \boldsymbol{b}+\frac{3}{8} \boldsymbol{c}$ & $\frac{1}{8} \boldsymbol{a}-\frac{1}{4} \boldsymbol{b}-\frac{1}{8} \boldsymbol{c}$ \\
\hline ORIGIN CHOICE 2 & $\Delta \mathbf{O}_{\text {IT Cell } 2}=-\frac{1}{4} \boldsymbol{a}+\frac{1}{4} \boldsymbol{b}$ & $\#$ 203: $\Delta \mathbf{O}_{\text {IT Cell } 2}=\frac{1}{4} \boldsymbol{a}-\frac{1}{4} \boldsymbol{b}$ \\
\hline \multicolumn{3}{|l|}{228} \\
\hline ORIGIN CHOICE 1 & $\Delta \mathbf{O}_{\text {IT Cell } 1}=-\frac{1}{8} \boldsymbol{a}+\frac{1}{4} \boldsymbol{b}+\frac{1}{8} \boldsymbol{c}$ & $\frac{3}{8} \boldsymbol{a}+\frac{1}{4} \boldsymbol{b}+\frac{1}{8} \boldsymbol{c}$ \\
\hline ORIGIN CHOICE 2 & $\Delta \mathbf{O}_{\text {IT Cell } 2}=-\frac{1}{4} \boldsymbol{a}+\frac{1}{4} \boldsymbol{b}$ & \\
\hline
\end{tabular}

lengths and (or) angles may not be changed. This is indicated by toolbar elements shown in gray.

\subsection{Space group and symmetry selection}

Figure 18 shows the interactive (hyperlink like) space group selection. Clicking blue text elements in the browser panel on the left of the GUI allows to access crystal systems, classes (point groups), and individual space groups.

Figure 19 illustrates the selection of symmetries from the complete list of Symmetries (left SGV GUI browser panel), which are present in the currently selected space group. Symmetries that are to be displayed can be selected according to their properties (angle, orientation, location, translation component). Several properties selected together will display only those symmetries that satisfy all properties. Another way is to open the generator list of a certain type of symmetry and select individual geometric algebra generators to be displayed (or to be removed from the display). 


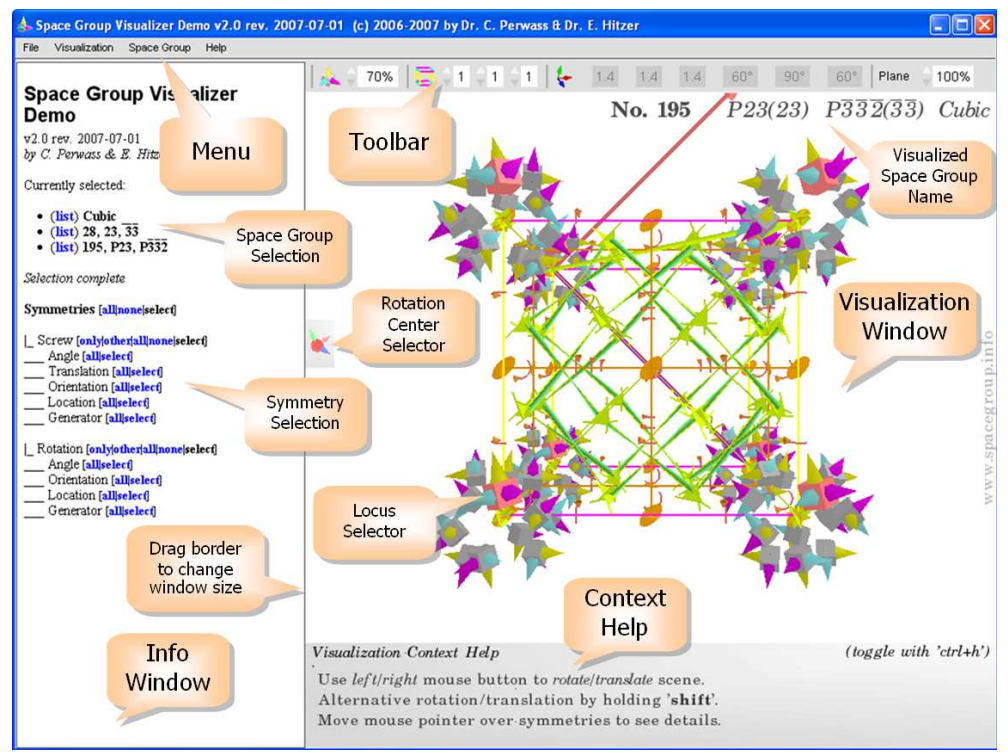

FIGURE 16. GUI of the Space Group Visualizer.

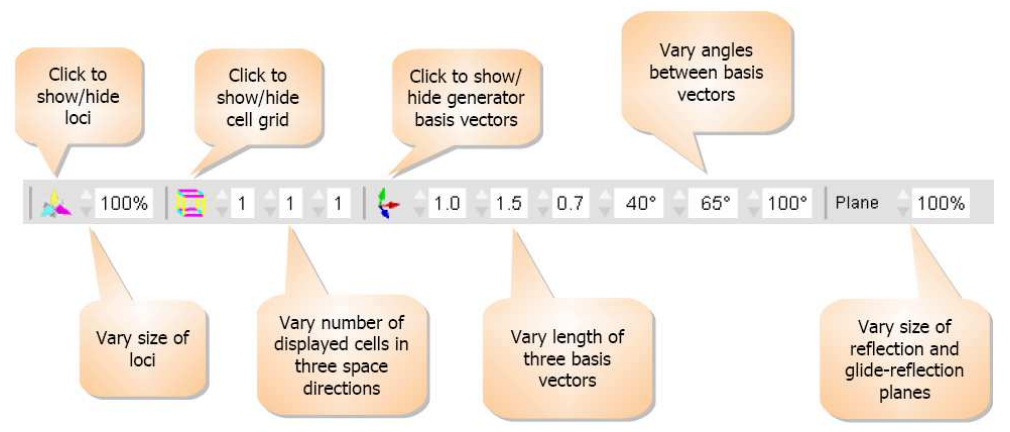

FIGURE 17. Toolbar of the Space Group Visualizer.

\subsection{Mouse pointer interactivity}

The mouse pointer allows a variety of visual interactions and animations, depending over which part of the visualization it is placed. Moving the mouse pointer over a symmetry element visualization both animates the symmetry and displays detailed information about this symmetry group element in the lower right corner. Animation means dynamic color and size changes; and the motion of general elements along a trajectory tracing the symmetry operation incrementally. Placement of the mouse pointer over a general element 


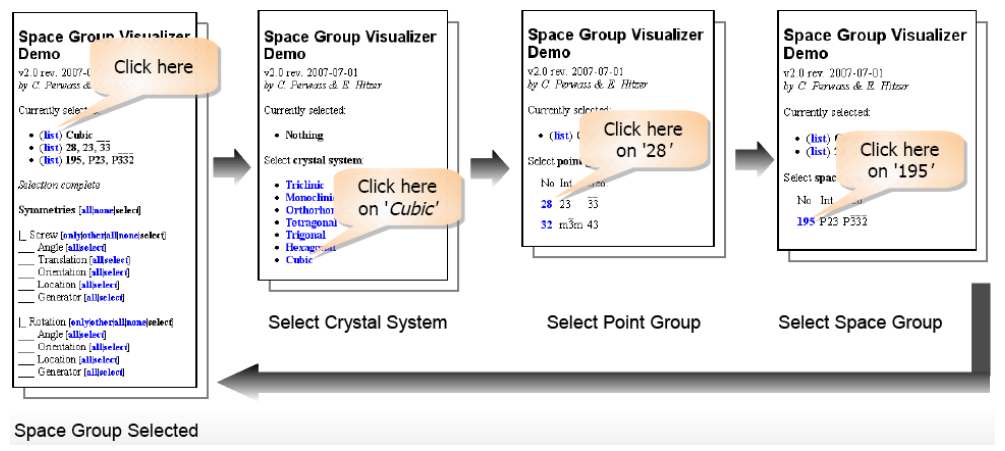

FIGURE 18. Space group selection from the Space Group Visualizer browser panel.

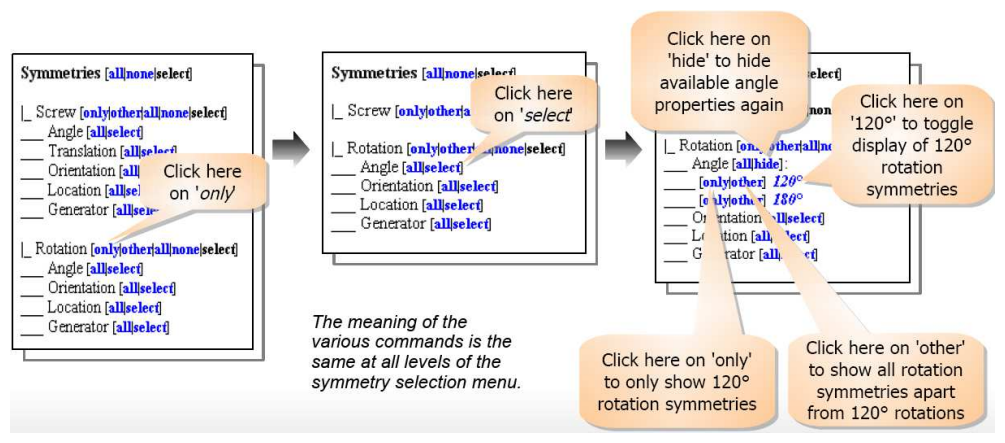

FIGURE 19. Space group selection of individual symmetries or groups of symmetries to be displayed.

(locus) selector activates it (blinking). The mouse pointer over the rotation center selector allows to change the rotation center of the mouse activated view rotation (described below).

The mouse pointer can be placed anywhere inside the visualization window. Holding down the left (right) mouse button and moving the mouse will rotate (translate) the visualization. Alternative rotation axes (translation directions) are activated by additionally holding the SHIFT key. With a 3D-mouse (3dconnexion) one can rotate and translate the view along all axes simultaneously. First placing the mouse pointer over a general element (locus) selector permits to translate and rotate it (together with all its symmetric partners). This provides an excellent way to grasp how one general element and the 3D symmetry represented in the space group determine the whole crystal structure.

A special feature of the SGV is the direct 3D graphics interaction. Simply placing the mouse pointer over a symmetry activates it and allows to: 

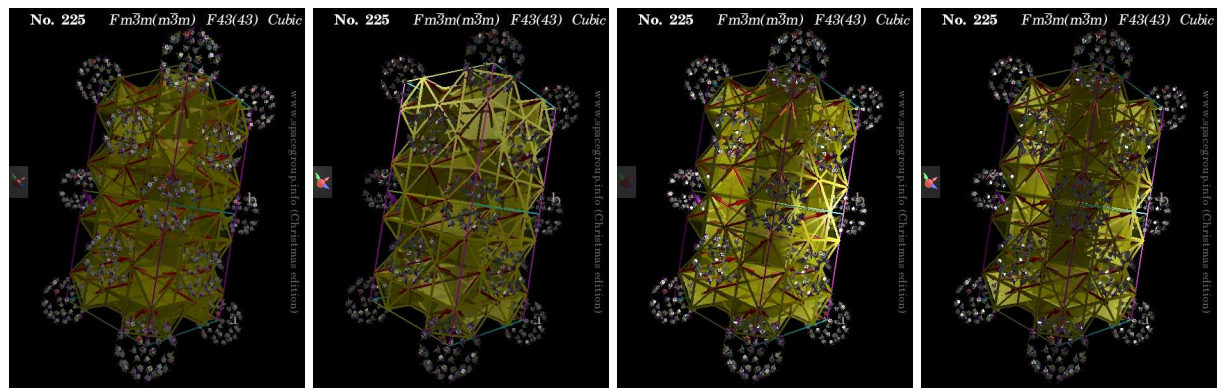

FIGURE 20. Examples of interactive SGV Visualization $>$ Lighting menu effects for cubic space group $F 43$ (Int. name $F m \overline{3} m$, No. 225). A cell bar of 2 cells with all glide reflection planes is shown, all other symmetries are deselected. From left to right: relative and central lighting off, relative lighting on, central lighting on, relative and central lighting on.

- Select only the activated symmetry (left mouse button). All other symmetries disappear from the view.

- Holding the CTRL key at the same time (while pressing the left mouse button) shows all symmetries (and only these) of the same type.

- Clicking the right mouse button removes an activated symmetry from the view.

- Holding the CTRL key at the same time (while pressing the right mouse button) removes all symmetries of the same type.

\subsection{Visualization options in detail}

The visualization drop down menu allows to toggle (activate and deactivate) the following visual functions

- Full screen mode.

- Orthographic view. The orthographic view allows the most direct comparison with ITA orthographic projections [11].

- Animation of the origin locus when a symmetry is activated (animated).

- Rotation animation of the whole view when it is pushed with the (left) mouse button.

- Reset the crystal view to visualizer default values.

- Reset general element (loci) positions.

The special visualization lighting menu provides a relative position light source. It is positioned relative to the visualization coordinate frame and moves with the visualization. Deselecting this option fixes the light source relative to the observer. The light source can optionally be positioned at the center of the coordinate frame, which is relative (or absolute) depending on the (de)selection of the relative position option, compare Fig. 20. The ambient light submenu allows to adjust the brightness of the ambient light, leading to more dramatic effects for darker settings. 


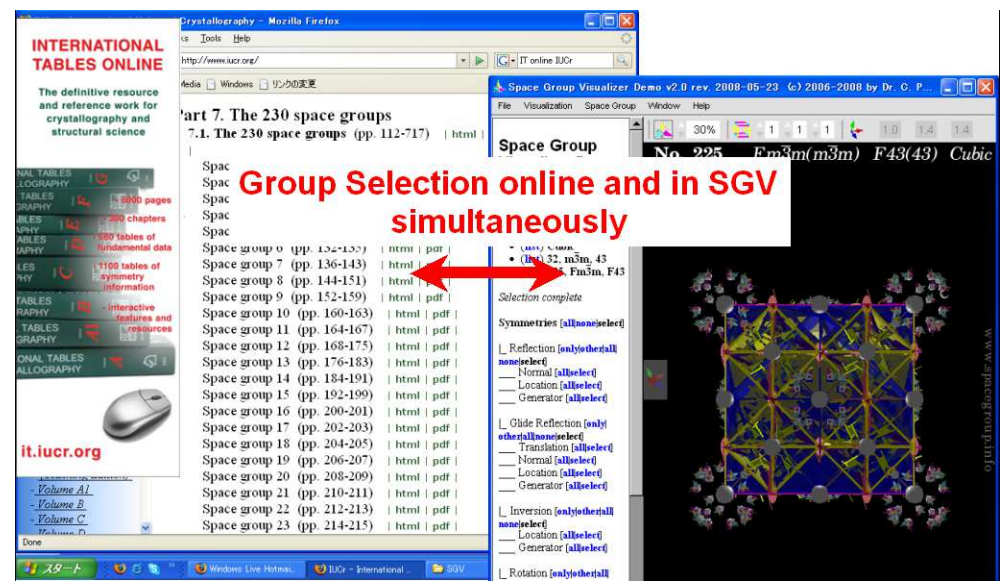

FIGURE 21. Parallel space group selection in the SGV window and the online ITA [11] space group window.

The color scheme menu item allows to select the current color scheme. For example a scheme with black background is more suitable for use in presentations, while a white background is better for publications, etc. It is possible via an XML file to individually define further color schemes. A color stereo option allows to specify cinema type stereo colors, which are best viewed with corresponding cinema color glasses in order to perform the full spatial 3D effect akin to virtual reality.

The cell type menu allows to select between different cell choices in the IT, Volume A [11], and (if different) a special geometric algebra type cell, which has the generating vectors $\boldsymbol{a}, \boldsymbol{b}, \boldsymbol{c}$ as cell axis attached to the cell origin.

\subsection{Integration with the online International Tables of Crystallography}

Through the window menu an additional window can be opened for displaying the pages of a space group from the online version of the International Tables of Crystallography, Volume A (ITA) [11]. For this the user must hold a valid user ID and password. When the online ITA can be accessed, the SGV and the online ITA window will always show the same space group. The user can synchronously navigate from space group to space group either in the SGV or in the online ITA window (cf. Fig. 21).

\subsection{Saving results}

It often takes some time to interactively bring the visualization into a desired state, which includes selection of a space group, selection and deselection of symmetry elements, 3D translations and rotations, adjustments of size, color and lighting, etc. If the user wants to save his work in order to show it to others, use it for teaching and presentations, produce publications, images for the internet, etc. the File menu offers the options to do a one step Quick Save View or a more detailed Save View. The latter will open a popup menu in order to choose the destination folder and the desired file name and file format. File 
formats available are: PNG (default), JPEG, Bitmap, Postscript, JPEG + EPS (get the same picture at once in two formats), and PNG with Bounding Box. The EPS option is particularly useful for use with Latex. In this way most of the figures were produced in this paper.

\section{Conclusion}

We have briefly reviewed the geometric algebra representation of $\mathbb{R}^{3}$ in the socalled conformal model and its use for the representation of $2 \mathrm{D}$ and $3 \mathrm{D}$ point groups and space groups. The key point is to only use physical crystal lattice vectors for the group generation.

Next we explained the relationship of the geometric symmetry vectors and cell origins of [14] to the conventional choices of cell vectors and origins of ITA2005. These relationships will certainly help crystallographers to familiarize themselves with the geometric algebra representation of space groups. Being able to convert the vectors of both descriptions back and forth also forms the basis of a future full scale conversion of geometric versor generators into $4 \times 4$ crystallographic matrix expressions and into the other conventional types of notation used in ITA2005, and vice versa.

The final part introduced the interactive software visualization of 3D space group symmetries based on the established geometric algebra representation. This implementation uses the conformal model both for generating the graphics itself and for internally computing with space group transformations.

Future options are the visualization of non-characteristic orbits of space group [28], subperiodic space groups, and magnetic space groups. The latter seems particularly attractive as it may nicely integrate the bivector representation of spin [12] in the real DiracHestenes equation of relativistic quantum physics. Based on CLUCalc a first rudimentary geometric algebra protein visualizer has been programmed recently for proteins of several thousand (up to 10 000) atoms. A possible future molecule (or ion group) toolbox may therefore be able to display complex biomolecule crystals as well.

\section{Acknowledgements}

E. Hitzer wishes to thank God: It (Jerusalem) shone with the glory of God, and its brilliance was like that of a very precious jewel, like a jasper, clear as crystal [29]. He thanks his family, his students D. Ichikawa, M. Sakai, K. Yamamoto, T. Ishii, his colleagues G. Sommer, D. Hestenes, J. Holt, T. Matsumoto, H. Wondratschek, M. Aroyo, H. Fuess, N. Ashcroft, and the organizers W. Rodrigues and J. Vaz of ICCA8 held in 2008 in Las Campinas, Brazil. We finally warmly thank the referee for his helpful comments and suggestions. 


\section{References}

[1] P. Angles, Construction de revetements du groupe conforme d'un espace vectoriel muni d'une metrique de type $(p, q)$, Ann. de l'I.H.P., Sect. A, Vol. 33, No. 1, 33-51 (1980).

[2] G. Aragón-González, J.L. Aragón, M.A. Rodríguez-Andrade and L. Verde-Star, Reflections, Rotations, and Pythagorean Numbers, Adv. appl. Clifford alg., Online First, 2008.

[3] E. Cartan, La Théorie des Spineurs I, II. Actualités Scientifiques et Industrielles, No. 643. Hermann, Paris, 1938.

[4] H.S.M. Coxeter, Discrete groups generated by reflections, Ann. of Math. 35, 588-621 (1934).

[5] H.S.M. Coxeter, W.O.J. Moser, Generators and Relations for Discrete Groups, Springer, 4th ed., 1980.

[6] J. Dieudonné, Sur les Groupes Classiques, Actualités Scientifiques et Industrielles, No. 1040. Hermann, Paris, 1948.

[7] C. Doran, A. Lasenby, Geometric Algebra for Physicists, CUP, Cambridge UK, 2003.

[8] A.W.M. Dress, T.F. Havel, Distance geometry and Geometric Algebra, Found. Phys., Vol. 23, No. 10, 1357-1374 (1993).

[9] J. Gallier, Geometric Methods and Applications, For Computer Science and Engineering, Springer, NY, 2001, chapter 7.

[10] J.D.M. Gutierrez, Operaciones de simitria mediante algebra geometrica aplicadas a grupos cristalograficos, Thesis, UNAM, Mexico, 1996.

[11] T. Hahn, Int. Tables of Crystallography A, Springer, Dordrecht, 2005; Online: it.iucr.org

[12] D. Hestenes, Spacetime Physics with Geometric Algebra, Am. J. Phys. Vol. 71, No. 7, 691-714 (2003).

[13] D. Hestenes, Point groups and space groups in geometric algebra, in L. Dorst et al (eds.), Applications of Geometric Algebra in Computer Science and Engineering, Birkhauser, Basel, 3-34 (2002);

[14] D. Hestenes, J. Holt, The crystallographic space groups in geometric algebra, J.M.P. Vol. 48, 023514 (2007).

[15] D. Hestenes, H. Li, A. Rockwood, New Algebraic Tools for Classical Geometry, in G. Sommer (ed.), Geometric Computing with Clifford Algebras, Springer, Berlin, pp. 4-26 (2001).

[16] E. Hitzer, Interactive and animated Geometric Algebra with Cinderella - Point Groups in Two Dimensions, http://sinai.mech.fukui-u.ac.jp/gcj/software/GAcindy-1.4/2dpointgroups.htm

[17] E. Hitzer, Euclidean Geometric Objects in the Clifford Geometric Algebra of \{Origin, 3-Space, Infinity\}, Bulletin of the Belgian Mathematical Society - Simon Stevin, Vol. 11, No. 5, 653662 (2004).

[18] E. Hitzer, Conic Sections and Meet Intersections in Geometric Algebra, Computer Algebra and Geometric Algebra with Applications, GIAE 2004, Revised Selected Papers, Springer, Lecture Notes in Computer Science, No. 3519, 350-362 (2005).

[19] E. Hitzer, C. Perwass, Crystal cells in geometric algebra, Proc. of Int. Symp. on Adv. Mech. Engrng (ISAME), Fukui, 290-295 (2004).

[20] E. Hitzer, C. Perwass, Full Geometric Description of All Symmetry Elements of Crystal Space Groups by the Suitable Choice of Only Three Vectors for Each Bravais Cell or Crystal Family, ISAME, Busan, 19-25 (2005). 
[21] C. Perwass, E. Hitzer, Interactive Visualization of Full Geometric Description of Crystal Space Groups, ISAME, Busan, 276-282 (2005).

[22] E. Hitzer, C. Perwass, Crystal Cell and Space Lattice Symmetries in Clifford Geometric Algebra, Int. Conf. on Numerical Analysis and Applied Mathematics, Rhodes, in T.E. Simos et al (eds.), Wiley-VCH, Weinheim, 937-941 (2005).

[23] E. Hitzer, C. Perwass, Three Vector Generation of Crystal Space Groups in Geometric Algebra, Bull. of the Soc. for Science on Form 21 55-6; Space Group Visualizer for Monoclinic Space Groups 38-9 (2006);

[24] C. Perwass, CLUCalc - a visual calculator, www.clucalc.info

[25] C. Perwass, Geometric Algebra with Applications in Engineering, Springer, NY, 2009.

[26] C. Perwass, E. Hitzer, Space Group Visualizer, www.spacegroup.info (2005).

[27] P. Lounesto, E. Latvamaa, Conformal Transformations and Clifford Algebras, Proc. of The AMS, Vol. 79, No. 4, 533-538 (1980).

[28] P. Engel, T. Matsumoto, G. Steinmann, H. Wondratschek, The non-characteristic orbits of the space groups, Z. Kristallogr., Supplement Issue No. 1. Raumgruppen-Projektionen. In Abhandlungen der Mathematisch-Physikalischen Klasse der Sächsischen Akademie, (1984).

[29] The Holy Bible, New International Version International Bible Society, Colorado Springs, 1984.

Eckhard Hitzer

Department of Applied Physics

University of Fukui

910-8507 Fukui

Japan

e-mail: hitzer@mech.fukui-u.ac.jp

Christian Perwass

Institute for Informatics

University of Kiel

24118 Kiel

Germany

e-mail: christianeperwass.de

Submitted: October 9, 2008

E. Hitzer, C. Perwass, Interactive 3D Space Group Visualization with CLUCalc and the Clifford Geometric Algebra Description of Space Groups, Adv. Appl. Clifford Alg., Vol. 20(3-4), pp. 631-658, (2010), DOI 10.1007/s00006-010-0214-z . 